\title{
Cooperative wireless energy harvesting and information transfer in stochastic networks
}

\author{
Chao Zhai ${ }^{1,2}$ and Ju Liu'1,2*
}

\begin{abstract}
In this paper, we consider a large-scale wireless ad hoc network with multiple source-destination communication pairs, where the sources operate with wireless energy harvesting. Before data transmission, each source should first harvest the radio frequency energy transferred from its corresponding destination. Since the source-destination distance is long, the efficiency of wireless energy transfer is very low. As a result, the transmission power of the source is weak, which is detrimental to the successful data transmissions. Instead, we introduce a relay in-between each source and destination for the wireless energy transfer and data transmission. The close distance between the relay and the source can improve the energy harvesting efficiency and the data relaying can improve the link robustness. With the assistance from the relay, the area spectrum efficiency is significantly enhanced compared with the non-cooperative system. A series of discrete power levels is defined for the sources and the probability of choosing each power level is analyzed by averaging over the random channel fading. We analyze the data success probabilities through averaging over the uncertain interference caused by the random locations of users, the channel fadings, and the various source transmission powers. The upper and lower approximations of data success probabilities are derived using the stochastic geometry theory for the cooperative energy and data transfer system. The optimal time allocation between the wireless energy transfer and the data transmission is investigated to maximize the system area throughput. Numerical and simulation results are provided to validate our theoretical analysis and show the dependence of system performance on various parameter settings. The results can provide the guidelines for the deployment of the energy harvesting cooperative communication system.
\end{abstract}

Keywords: Energy harvesting; Cooperative diversity; Stochastic geometry; Interference channel; Success probability; Area throughput

\section{Introduction}

Wireless ad hoc or sensor networks are often composed of small terminals, which are deployed in the extreme environment to realize particular monitoring and communication functions. For example, the small sensors can be embedded inside the body of a human to monitor the health conditions for medical treatment. The limited capacity of the small battery is a major bottleneck impeding the long-time operation of the wireless network. It is difficult or impossible to recharge or replace the small batteries due to the untouchable deployment of terminals. To make the network sustainable, it becomes much

\footnotetext{
*Correspondence: juliu@sdu.edu.cn

${ }^{1}$ School of Information Science and Engineering, Shandong University, 27 Shanda Nanlu, 250100 Jinan, China

${ }^{2}$ National Mobile Communications Research Laboratory, Southeast University, 2 Sipailou, 210096 Nanjing, China
}

attractive if each small terminal can self-recharge its battery through harvesting the energy from the environment. Practically, the energy can be harvested from the solar and the kinetic activities, such as wind, water, pressure, and vibration [1], but the energy harvesting $(\mathrm{EH})$ often occurs sporadically and intermittently. To cope with the uncertainty of $\mathrm{EH}$ and efficiently utilize the harvested energy, the optimal transmission policy with the format of directional water filling is designed in [2] and [3] under the energy causality constraint, which means the energy can only be used after it has been harvested. Since the energy is harvested and stored in the battery or super-capacitor, there are some practical issues in the design of the optimal transmission strategy, such as EH efficiency, battery capacity, and battery leakage [4].

Apart from the above EH sources, the energy can also be harvested from the electromagnetic radiations. It is a

\section{Springer}

(c) 2015 Zhai and Liu; licensee Springer. This is an Open Access article distributed under the terms of the Creative Commons Attribution License (http://creativecommons.org/licenses/by/4.0), which permits unrestricted use, distribution, and reproduction in any medium, provided the original work is properly credited. 
promising direction to harvest the radio frequency (RF) energy, as plenty of microwave signals are surrounding us nearly anywhere at anytime. The RF can carry not only the information but also the energy, as the rectifying antenna can help the receiver intercept the energy signal. If the receiver operates with $\mathrm{EH}$, both the time-switching and the power-splitting methods can be adopted to separate the RF signal for the $\mathrm{EH}$ and information decoding (ID) [5]. The efficiency of wireless energy transfer (WET) is strictly related with the distance between the transmitter and the receiver, because the RF signal suffers from severe path-loss over the long transmission distance. Researchers have shown that using the frequency band of $915 \mathrm{MHz}$, an energy amount of $3.5 \mathrm{~mW}$ can be harvested over the short distance of $0.6 \mathrm{~m}$, while the $\mathrm{EH}$ amount is merely $1 \mu \mathrm{W}$ for the long distance of $11 \mathrm{~m} \mathrm{[6].} \mathrm{Therefore,} \mathrm{it} \mathrm{is} \mathrm{more} \mathrm{bene-}$ ficial to wirelessly transfer energy over a short distance to improve the EH efficiency at the receiver side. In general, $\mathrm{EH}$ is an environment-friendly technique to significantly prolong the lifetime of wireless networks with no carbon emissions.

Liu et al. studied the opportunistic RF EH over the point-to-point interference link, where the receiver can adaptively switch between EH and ID based on the observations of signal and interference strength, and the optimal switching rule is determined to achieve the trade-off between the EH amount and the ID capacity [7]. In the multiple-input multiple-output (MIMO) broadcast system with one access point (AP) serving two user terminals (UTs), the optimal transmission strategy with simultaneous wireless information and power transfer (SWIPT) from the AP is proposed in [8], and the trade-off between the information rate and the energy transfer is achieved by scheduling each UT either for the EH or ID. The power control mechanism based on the WET is further studied in the broadband system with orthogonal frequency division multiplexing (OFDM), where the optimal beamforming is developed at the base station (BS) to create a set of parallel sub-channels for the SWIPT [9]. Furthermore, Ju et al. proposed the optimal time allocation between EH and data transmission to maximize the sum throughput of the multiuser system, where an AP can wirelessly transfer energy to the UTs over the downlink and, using the harvested energy the UTs, can successively transmit their information to the AP over the uplink [10].

As well known, cooperative relaying can introduce the space diversity and thereby improve the robustness of wireless data transmissions. The advantage of cooperation can also be utilized to improve the energy and spectrum efficiency of wireless EH networks. In the traditional source-relay-destination cooperative system with the relay powered by the RF EH, Nasir et al. investigated the optimal resource allocation between $\mathrm{EH}$ and data relaying to maximize the system throughput [11].
If there are multiple source-destination communication pairs assisted by a common RF EH relay with the energy transferred from sources, Ding et al. studied how to efficiently distribute the harvested energy at the relay to facilitate the data transmissions over multiple links [12]. Based on the power-splitting method, Krikidis studied the performance of the simultaneous information and energy transfer with/without relaying [13] in the stochastic network. Since the data traffic and the energy status of two neighboring cellular systems are different, the joint energy and spectrum cooperation approach is developed by considering the inter-system resource complementarity [14]. Huang et al. studied the trade-offs between system parameters in the hybrid network with data BSs and power BSs overlaid on the two-dimensional (2-D) plane [15]. Given the EH rate, the transmission probability is analyzed in [16] for each mobile terminal considering its battery capacity, based on which the maximal throughput of the wireless network is derived. In the underlay cognitive radio (CR) network, the guard zones are applied around each primary link [17] to protect the transmission quality of primary users, within which the secondary users should keep silent but they can harvest the wireless energy radiated from the primary transmitter [18]. The system performance is analyzed for the large-scale overlay $C R$ network [19], where the secondary users can help the primary data transmission to exchange for the opportunity of spectrum sharing.

In this paper, we consider a large-scale wireless $a d$ hoc network where the source-destination pairs are assumed to be randomly distributed over the whole plane. Each source has a corresponding destination, while a relay inbetween each source-destination pair is activated for the energy and data cooperations. The source operates with wireless $\mathrm{EH}$, while all the other terminals have the continuous power supply. Each time block is divided into two phases, one is for the WET from the destination and relay to the source, another is for the data transmission from the source and relay to the destination. In the beginning of each time block, the destination should first wirelessly transfer energy to the source alone or together with the relay. Using the harvested energy, the source can transmit its data to the destination with assistance from the relay according to the decode-and-forward (DF) incremental relaying protocol [20]. The main contributions of our paper are summarized as follows.

- We propose the relay-based cooperative WET and data transmission framework by taking into account the spatial randomness of users. The assistance from the relay can help improve both the EH efficiency and the data transmission robustness.

- Since the source harvests energy from the destination and the relay over the fading channels, the energy 
amount available for the data transmission is various across different links. We define a group of discrete power levels for each source and analyze the probability of choosing each power level for the data transmission.

- Considering the various transmission powers of different sources and taking into account the uncertainty of interference, we analyze the data success probabilities with or without relaying using the stochastic geometry theory. The upper and lower approximations of success probabilities are derived for the cooperative system.

- Based on the data success probabilities, the optimal time allocation between the wireless $\mathrm{EH}$ and data transmission is investigated through maximizing the area throughput of both the non-cooperative and cooperative systems. The performance results show the impacts of various parameter configurations to the system performance.

The rest of this paper is organized as follows. In Section 2, we introduce the system model about the noncooperative and cooperative wireless energy and data transfer. Section 3 sets the transmission powers and studies the probability of choosing each power level. Section 4 analyzes the data success probabilities and area throughput of the non-cooperative link. The performance of the cooperative link is studied in Section 5. Numerical and simulation results are presented in Section 6. Section 7 concludes this paper.

\section{System model}

We consider a large-scale wireless ad hoc network as shown in Figure 1, where each source node has a corresponding destination node. The distance between each source and its destination is assumed to be a constant $d$. In fact, the relaxed assumption about random distance cannot bring us much insight but complicates the performance analysis [21]. The source nodes are assumed to be distributed on the 2-D plane following homogeneous Poisson point process (PPP) $\Pi_{\mathrm{s}}=\left\{x_{i}, i \in \mathbb{Z}\right\}$ with density $\lambda_{s}$. A relay network is placed randomly in the geographic region of the wireless ad hoc network to facilitate the energy and data cooperation. The density of the relay nodes is assumed to be much higher than that of the source nodes. The source node operates with the RF EH, while each relay and destination is assumed to have continuous and stable power supplies, e.g., they may connect to the power grid or have a large-capacity battery. At a particular location inbetween each source-destination pair, a relay is selected for the cooperation, while the unselected relay nodes may transmit their own data without interfering the source links.
By modeling the locations of nodes as a PPP in $\mathbb{R}^{2}$ with density $\lambda>0$, we have the following two characteristics [17,22]: i) for every bounded closed set $B \subset \mathbb{R}^{2}$, the counting measure (number of nodes) $\Phi(B)$ follows the Poisson distribution with mean $\lambda|B|$, where $|B|$ denotes the area of $B$. So, the probability density function can be given as $\operatorname{Pr}\{\Phi(B)=k\}=e^{-\lambda|B|} \frac{(\lambda|B|)^{k}}{k !}$. ii) If $B_{1}, \ldots, B_{m}$ are disjoint sets, then the counting measures $\Phi\left(B_{1}\right), \ldots, \Phi\left(B_{m}\right)$ are independent random variables. Given a particular realization of the number of nodes as $\Phi(B)=k$, then the $k$ nodes are independently, uniformly distributed in the area $B$. The PPP has been widely adopted to model the randomness of wireless networks, based on which the network performance can be analyzed according to the Campbell's theorem, the probability generating function (PGFL), and the Slivnyak theorem [22].

In the non-cooperative scenario, each destination should first wirelessly transfer energy to its source over the reverse link, then using the harvested energy, the source transmits its data to the destination over the forward link. However, for the relay-based scenario, both the relay and the destination can simultaneously transmit the microwave to recharge the battery of the source. Afterwards, the relay can actively help the data transmission from the source to the destination. Since the relay is much closer to the source than the destination, the amount of energy harvested by the source can be boosted with the cooperation from the relay. Meanwhile, the space diversity can be expected to improve the system performance with the cooperative data relaying. Since the relay nodes have a much higher density than the source nodes, it is appropriate to assume that always a relay node can be found at a particular location between each source and its destination.

The source is assumed to always have the data intended for the destination. The whole duration of data transmission is divided into successive equal-length time blocks. The channel fading keeps constant in each time block, but it changes independently from one block to another. The channel fading is assumed to be independent across different communication links. The time-switching technique is adopted between the source and the destination to realize the wireless energy and information transfer successively.

- For the non-cooperative system, the source should firstly harvest the wireless energy from its destination in a fraction of each time block. Then, using the harvested energy, the source transmits its data to the destination.

- For the cooperative system, the source should first harvest the wireless energy transferred from both the destination and the relay. After the wireless $\mathrm{EH}$, a 


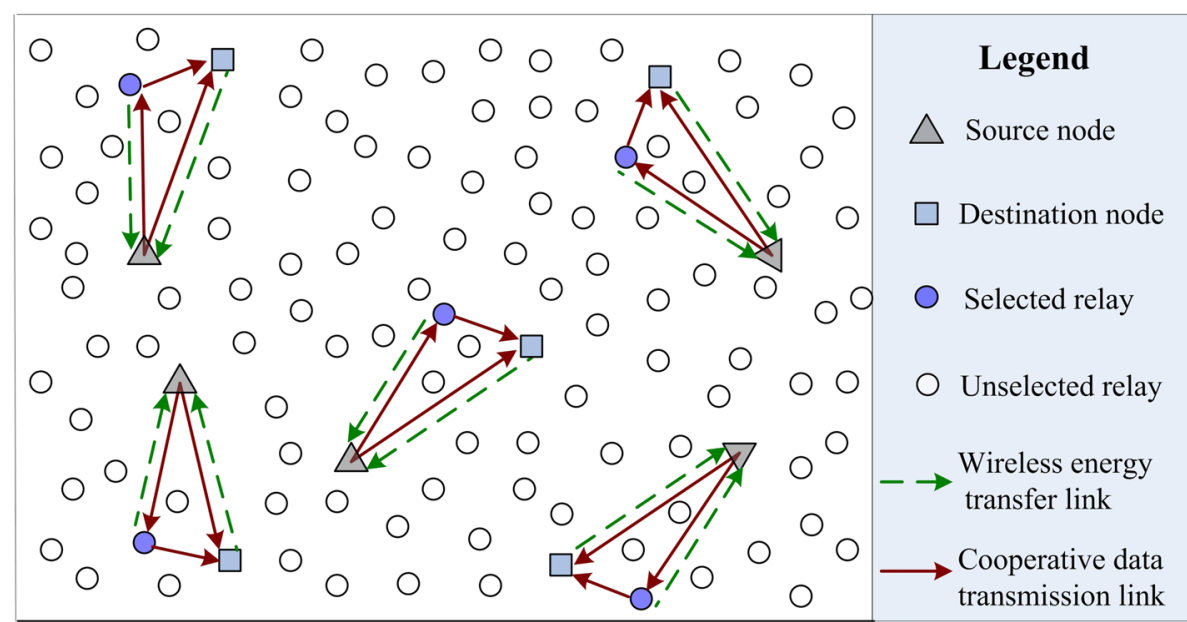

Figure 1 System model. There are multiple source-destination links randomly distributed over the whole plane. The relay nodes are more densely distributed, and one relay is selected at a particular location to cooperatively transfer energy and data for each source-destination pair.

data packet is transmitted from the source to its destination with the assistance from the relay.

In the sequel, the non-cooperative system is studied to serve as a benchmark to show the advantage of the cooperative scheme. The difference between the non-cooperative and cooperative systems is compared in Table 1. The success probabilities of non-cooperative and cooperative data transmissions will be analyzed by properly modeling the mutual interference between concurrent links. Furthermore, the time allocation between $\mathrm{EH}$ and data transmission will be optimized to maximize the network area throughput.

Table 1 Comparison between the non-cooperative and cooperative WET and data transmission for each source-destination pair in a time block

\begin{tabular}{|c|c|c|}
\hline & $\begin{array}{l}\text { Non-cooperative } \\
\text { link }\end{array}$ & $\begin{array}{l}\text { Cooperative } \\
\text { link }\end{array}$ \\
\hline $\begin{array}{l}\text { Which relay } \\
\text { is selected? }\end{array}$ & $\begin{array}{l}\text { No relay } \\
\text { selection }\end{array}$ & $\begin{array}{l}\text { Relay at a } \\
\text { particular location }\end{array}$ \\
\hline $\begin{array}{l}\text { Time fraction } \\
\text { for the WET }\end{array}$ & $\tau$ & $\tau$ \\
\hline $\begin{array}{l}\text { Wireless energy } \\
\text { transfer }\end{array}$ & Destination $\rightarrow$ source & $\begin{array}{l}\text { Destination and } \\
\text { relay } \rightarrow \text { source }\end{array}$ \\
\hline $\begin{array}{l}\text { Data transmission } \\
\text { duration }\end{array}$ & $\frac{1-\tau}{2}$ & $\frac{1-\tau}{2}$ \\
\hline Data transmission & Source $\rightarrow$ destination & Source $\rightarrow$ destination \\
\hline $\begin{array}{l}\text { Data retransmission } \\
\text { duration }\end{array}$ & $\frac{1-\tau}{2}$ & $\frac{1-\tau}{2}$ \\
\hline Data retransmission & Source $\rightarrow$ destination & $\begin{array}{l}\text { Source or } \\
\text { relay } \rightarrow \text { destination }\end{array}$ \\
\hline
\end{tabular}

\section{Transmission power settings}

In each time block, the wireless energy is first harvested and stored in the battery of the source, then the source transmits its data using a certain power determined by the $\mathrm{EH}$ amount. We define a series of discrete power levels and each source should choose one power for its data transmission. The number of power levels is set as $N+1$, and the peak transmission power of each source is denoted as $\hat{p}$. The power levels are defined as $\left\{p_{0}=0, p_{1}=\frac{\hat{p}}{N}, p_{2}=\frac{2 \hat{p}}{N}, \ldots, p_{N-1}=\frac{(N-1) \hat{p}}{N}, p_{N}=\hat{p}\right\}$, where the power zero represents no data transmission. Specifically, the discrete power becomes continuous with $N \rightarrow \infty$, and there is only one available power with $N=1$. Since the link for the WET suffers from independent channel fading, the $\mathrm{EH}$ amount at each source is random and hence the transmission power varies for different sources. Therefore, the general discrete power setting can reflect the imbalance of the $\mathrm{EH}$ amount for different source nodes. Since the locations of the source nodes with the same transmission power can be viewed as a homogeneous PPP, the performance analysis can be greatly simplified by dividing the power into discrete levels.

\subsection{Power setting for the non-cooperative system}

In the non-relay scenario, the WET is performed by each destination in the first $\tau$ fraction of each time block. In this case, the energy harvested by a typical source node is expressed as [11]:

$$
\mathcal{Q}_{o}^{\mathrm{n}}=\eta \tau p_{\mathrm{e}} \tilde{G}_{o} \min \left(1, d^{-\alpha}\right)
$$


where $\eta$ represents the EH efficiency, $p_{\mathrm{e}}$ is the power of the destination for the WET, $\tilde{G}_{o}$ is the channel fading of the typical link for the energy transfer, and $d$ is the source-destination distance. The interference from other concurrent links and the additive noise are not incorporated in the EH model as assumed in [18]. This assumption can lead to the succinct result of the system performance using the stochastic geometry theory, and it has minor impacts to the $\mathrm{EH}$ result. On the one hand, the power of interference plus noise is relatively weak due to the sparse distribution of sources. If multiple antennas are equipped at each destination, the WET can be performed using the beamforming method by directing the main beam to the intended source, which can further reduce the interference between links. On the other hand, the sensitivity of the energy receiver is much lower compared with the information receiver. Therefore, the interference affects the ID more severely than the EH. To avoid the impractical power amplification over the wireless medium, the short-range path-loss model is adopted, which is defined as the minimal value between one and the distance raised by $(-\alpha)$ with $\alpha$ being the path-loss exponent. The channel fading for the WET is assumed to be independent with that for the information transmission, because disjoint bandwidths are used in the two processes.

Given the EH amount in the $\tau$ fraction of a time block, the maximal continuous transmission power in the remaining $1-\tau$ time fraction is denoted as $\tilde{p}_{o}$ for the typical link. For the pairwise power control, the total consumed energy in each block should equal the total harvested energy, i.e., $(1-\tau)\left(\tilde{p}_{o}+p_{\mathrm{c}}\right)=\mathcal{Q}_{o}^{\mathrm{n}}$, where $p_{\mathrm{c}}$ is a constant representing the circuit power consumption. We consider the circuit power, because when the communication distance is short and the transmission power is weak, the circuit power may dominate the whole power consumption [23]. The maximal continuous power can be obtained as:

$$
\tilde{p}_{o}=\frac{\mathcal{Q}_{o}^{\mathrm{n}}}{1-\tau}-p_{\mathrm{c}}=\left(\frac{\tau}{1-\tau}\right) \eta p_{\mathrm{e}} \tilde{G}_{o} \min \left(1, d^{-\alpha}\right)-p_{\mathrm{c}}
$$

which may be even less than zero. It can be seen from Equation 2 that the continuous transmission power of the source is a function of the time allocation factor $\tau$. For any other active source node $x \in \Pi_{\mathrm{s}}$, we denote the EH amount and the maximal power as $\mathcal{Q}_{x}^{\mathrm{n}}$ and $\tilde{p}_{x}$, respectively.

Next, we will analyze the probability of choosing each power level through averaging over the channel fading between the destination and the source. The probability of choosing power level $p_{i}$ with $i \in\{1, \ldots, N-1\}$ for the data transmission is denoted as $\delta_{i}=\operatorname{Pr}\left\{p_{i} \leq \tilde{p}_{o}<p_{i+1}\right\}$ and calculated as:

$$
\begin{aligned}
\delta_{i} & =\operatorname{Pr}\left\{\frac{(1-\tau)\left(p_{i}+p_{\mathrm{c}}\right)}{\eta \tau p_{\mathrm{e}} \min \left(1, d^{-\alpha}\right)} \leq \tilde{G}_{o}<\frac{(1-\tau)\left(p_{i+1}+p_{\mathrm{c}}\right)}{\eta \tau p_{\mathrm{e}} \min \left(1, d^{-\alpha}\right)}\right\} \\
& =\exp \left[-\frac{(1-\tau)\left(p_{i}+p_{\mathrm{c}}\right)}{\eta \tau p_{\mathrm{e}} \min \left(1, d^{-\alpha}\right)}\right]-\exp \left[-\frac{(1-\tau)\left(p_{i+1}+p_{\mathrm{c}}\right)}{\eta \tau p_{\mathrm{e}} \min \left(1, d^{-\alpha}\right)}\right]
\end{aligned}
$$

The probability is derived based on the assumption that the channel power fading is exponentially distributed with unit mean, which is true for the Rayleigh fading. We can see from (3) that the probability of choosing power level $p_{i}$ is dependent on the time allocation factor $\tau$. Given the other system parameters, the maximal probability of choosing $p_{i}$ can be calculated by setting the derivative of $\delta_{i}$ with respect to $\tau$ as zero. The time fraction $\tau_{i}$ that can maximize the probability of choosing power level $p_{i}$ can thus be derived as:

$$
\tau_{i}=\frac{p_{i+1}-p_{i}}{\left(p_{i+1}-p_{i}\right)+\eta p_{\mathrm{e}} \min \left(1, d^{-\alpha}\right) \ln \left(\frac{p_{i+1}+p_{\mathrm{c}}}{p_{i}+p_{\mathrm{c}}}\right)} .
$$

Substitute the optimal value of $\tau_{i}$ into the expression of $\delta_{i}$, we can obtain the maximal probability of choosing the power level $p_{i}$ as:

$$
\begin{aligned}
\delta_{i}^{\star}= & \exp \left[-\left(\frac{p_{i}+p_{\mathrm{c}}}{p_{i+1}-p_{i}}\right) \ln \left(\frac{p_{i+1}+p_{\mathrm{c}}}{p_{i}+p_{\mathrm{c}}}\right)\right] \\
& -\exp \left[-\left(\frac{p_{i+1}+p_{\mathrm{c}}}{p_{i+1}-p_{i}}\right) \ln \left(\frac{p_{i+1}+p_{\mathrm{c}}}{p_{i}+p_{\mathrm{c}}}\right)\right],
\end{aligned}
$$

where $\ln (\cdot)$ is the natural logarithm.

Particularly, the probability of choosing the power level $p_{0}=0$ is expressed as $\delta_{0}=\operatorname{Pr}\left\{\tilde{p}_{o}<p_{1}\right\}$ and it can be calculated as:

$$
\delta_{0}=1-\exp \left[-\frac{(1-\tau)\left(p_{1}+p_{\mathrm{c}}\right)}{\eta \tau p_{\mathrm{e}} \min \left(1, d^{-\alpha}\right)}\right]
$$

The partial derivative of $\delta_{0}$ with respect to $\tau$ is obtained as:

$$
\frac{\partial \delta_{0}}{\partial \tau}=-\frac{p_{1}+p_{\mathrm{c}}}{\eta \tau^{2} p_{\mathrm{e}} \min \left(1, d^{-\alpha}\right)} \exp \left[-\frac{(1-\tau)\left(p_{1}+p_{\mathrm{c}}\right)}{\eta \tau p_{\mathrm{e}} \min \left(1, d^{-\alpha}\right)}\right],
$$

which is always less than zero, so the probability of the source keeping silent is a monotonically decreasing function of the parameter $\tau$. 
Moreover, the probability of choosing the maximal power level $p_{N}$ is expressed as $\delta_{N}=\operatorname{Pr}\left\{\tilde{p}_{o} \geq p_{N}\right\}$ and it can be obtained as:

$$
\delta_{N}=\exp \left[-\frac{(1-\tau)\left(p_{N}+p_{\mathrm{c}}\right)}{\eta \tau p_{\mathrm{e}} \min \left(1, d^{-\alpha}\right)}\right] .
$$

The partial derivative of $\delta_{N}$ with respect to $\tau$ is obtained as:

$$
\frac{\partial \delta_{N}}{\partial \tau}=\frac{p_{N}+p_{\mathrm{c}}}{\eta \tau^{2} p_{\mathrm{e}} \min \left(1, d^{-\alpha}\right)} \exp \left[-\frac{(1-\tau)\left(p_{N}+p_{\mathrm{c}}\right)}{\eta \tau p_{\mathrm{e}} \min \left(1, d^{-\alpha}\right)}\right],
$$

where we can see that the partial derivative is always greater than zero with $0<\tau<1$, which means the probability of choosing power level $p_{N}$ is a monotonically increasing function of the time allocation factor $\tau$. When $\tau$ approaches one, the probability $\delta_{N}$ becomes almost one, and this phenomenon is independent with other parameters. With the increase of the time factor $\tau$, more energy is harvested and shorter time is reserved for the data transmission, which results in a much higher transmission power.

Figure 2 shows the probability of choosing a power level with respect to the time allocation factor $\tau$. There are six power levels by setting $N=5$. Without specifying otherwise, the peak transmission power is set as $\hat{p}=0.1 \mathrm{w}$, the distance between a source and its destination is $d=10$ $\mathrm{m}$, the energy transfer power of the destination is $p_{\mathrm{e}}=15$ $\mathrm{dB}$, the circuit power consumption is $p_{\mathrm{c}}=0.01 \mathrm{w}$, the $\mathrm{EH}$ efficiency is $\eta=0.8$, and the path-loss exponent is $\alpha=3$.
It can be seen from Figure 2 that the probability of choosing a non-zero and non-peak power level first increases and then decreases when the EH time is prolonged. In the lower regime of $\tau$, more energy is harvested with the increase of $\tau$, so the probability of choosing a power level gets larger. However, with the further increase of $\tau$ in the high regime, it is more likely to choose a higher power level, so the probability of choosing the lower power level gets smaller. The maximal probability of choosing each power level has been obtained in (5), and it is plotted in this figure. Moreover, it verifies that the probability of choosing the zero/peak transmission power monotonically decreases/increases with more time allocated for the WET.

\subsection{Power setting for the cooperative system}

For the cooperative WET, in the first $\tau$ fraction of each time block, the relay and the destination can simultaneously transfer the RF energy to the source. The longer the distance for the WET, the more severe the impact from the path-loss, which can greatly attenuate the energy amount arriving at the source. As the distance between the relay and the source is much shorter than the distance between the destination and the source, the efficiency of WET jointly performed by the relay and the destination is much higher than that performed by the destination alone. In the EH period, the total amount of energy recharged into the battery of the source is modeled as:

$$
\mathcal{Q}_{o}^{\mathrm{c}}=\eta \tau p_{\mathrm{e}}\left[\tilde{G}_{r} \min \left(1, \tilde{d}^{-\alpha}\right)+\tilde{G}_{s} \min \left(1, d^{-\alpha}\right)\right],
$$

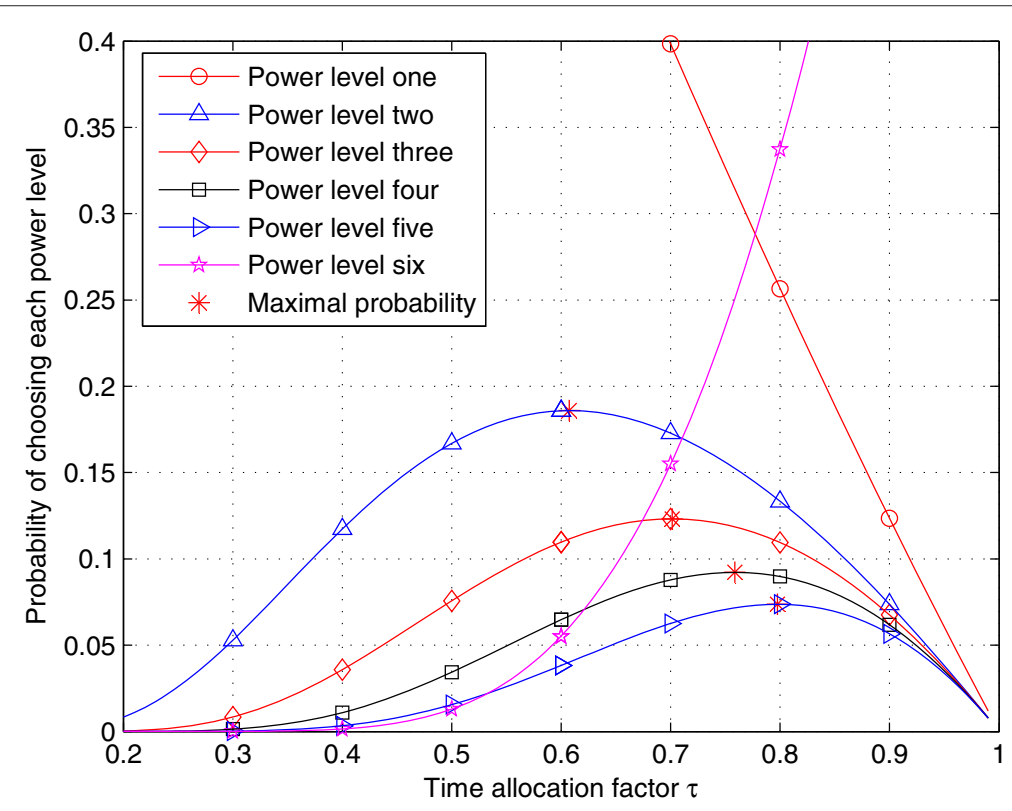

Figure 2 Probability of choosing each power level w.r.t. the time allocation factor $\tau$ for the noncooperative WET. 
where $\tilde{d}=\beta d$ with $0<\beta<1$ represents the distance between the relay and the source. The energy transfer power of the relay is the same as the destination and it is denoted as $p_{\mathrm{e}}$. The channel fading of WET between the relay and the source is denoted as $\tilde{G}_{r}$, while the channel fading of WET between the destination and the source is denoted as $\tilde{G}_{s}$. The two random variables of channel fading over the two different links towards the source are independent.

After the wireless $\mathrm{EH}$ in the first fraction $\tau$ of each time block, the source data is cooperatively transmitted to the destination in the remaining time fraction $1-\tau$. The pairwise power control is considered, where the consumed energy equals the amount of harvested energy, that is, $\left(\tilde{p}_{o}+p_{\mathrm{c}}\right)(1-\tau)=\mathcal{Q}_{o}^{\mathrm{c}}$. In a certain time block, the maximal continuous transmission power of the source can be obtained as:

$$
\begin{aligned}
\tilde{p}_{o} & =\frac{\mathcal{Q}_{o}^{\mathrm{c}}}{1-\tau}-p_{\mathrm{c}} \\
& =\left(\frac{\tau}{1-\tau}\right) \eta p_{\mathrm{e}}\left[\tilde{G}_{r} \min \left(1, \tilde{d}^{-\alpha}\right)+\tilde{G}_{s} \min \left(1, d^{-\alpha}\right)\right]-p_{\mathrm{c}} .
\end{aligned}
$$

According to the divisions of the power levels in the previous subsection, the discrete transmission power can be set as $p_{o}$. The probability of choosing the non-zero and non-peak power level $p_{i}(i \in\{1, \ldots, N-1\})$ for the cooperative data transmission is expressed as $\mu_{i}=$ $\operatorname{Pr}\left\{p_{i} \leq \tilde{p}_{o}<p_{i+1}\right\}$, and it is calculated as:

$$
\begin{aligned}
\mu_{i}= & \operatorname{Pr}\left\{\frac{(1-\tau)\left(p_{i}+p_{\mathrm{c}}\right)}{\eta \tau p_{\mathrm{e}}}-\tilde{G}_{r} \min \left(1, \tilde{d}^{-\alpha}\right)\right. \\
& \leq \tilde{G}_{s} \min \left(1, d^{-\alpha}\right)<\frac{(1-\tau)\left(p_{i+1}+p_{\mathrm{c}}\right)}{\eta \tau p_{\mathrm{e}}} \\
& \left.-\tilde{G}_{r} \min \left(1, \tilde{d}^{-\alpha}\right)\right\} .
\end{aligned}
$$

It can be seen from (12) that the probability of choosing a certain power level is calculated by averaging over two independent exponential random variables. The probability of (12) can be decomposed into two probabilities according to the value of $\tilde{G}_{r}$, that is, $\mu_{i}=\mu_{i 1}+\mu_{i 2}$. The first component probability $\mu_{i 1}$ is given as:

$$
\begin{aligned}
\mu_{i 1}= & \operatorname{Pr}\left\{\tilde{G}_{s}<\frac{(1-\tau)\left(p_{i+1}+p_{\mathrm{c}}\right)}{\eta \tau p_{\mathrm{e}} \min \left(1, d^{-\alpha}\right)}-\frac{\tilde{G}_{r} \min \left(1, \tilde{d}^{-\alpha}\right)}{\min \left(1, d^{-\alpha}\right)},\right. \\
& \left.\frac{(1-\tau)\left(p_{i}+p_{\mathrm{c}}\right)}{\eta \tau p_{\mathrm{e}} \min \left(1, \tilde{d}^{-\alpha}\right)} \leq \tilde{G}_{r}<\frac{(1-\tau)\left(p_{i+1}+p_{\mathrm{c}}\right)}{\eta \tau p_{\mathrm{e}} \min \left(1, \tilde{d}^{-\alpha}\right)}\right\} .
\end{aligned}
$$

The second component probability $\mu_{i 2}$ is given as:

$$
\begin{aligned}
\mu_{i 2}= & \operatorname{Pr}\left\{\frac{(1-\tau)\left(p_{i}+p_{\mathrm{c}}\right)}{\eta \tau p_{\mathrm{e}} \min \left(1, d^{-\alpha}\right)}-\frac{\tilde{G}_{r} \min \left(1, \tilde{d}^{-\alpha}\right)}{\min \left(1, d^{-\alpha}\right)}\right. \\
& \leq \tilde{G}_{s}<\frac{(1-\tau)\left(p_{i+1}+p_{\mathrm{c}}\right)}{\eta \tau p_{\mathrm{e}} \min \left(1, d^{-\alpha}\right)}-\frac{\tilde{G}_{r} \min \left(1, \tilde{d}^{-\alpha}\right)}{\min \left(1, d^{-\alpha}\right)}, \\
& \left.\tilde{G}_{r}<\frac{(1-\tau)\left(p_{i}+p_{\mathrm{c}}\right)}{\eta \tau p_{\mathrm{e}} \min \left(1, \tilde{d}^{-\alpha}\right)}\right\} .
\end{aligned}
$$

Take the expectations over the two channel fading variables, the probabilities $\mu_{i 1}$ and $\mu_{i 2}$ can be derived in the following two propositions.

Proposition 1. The probability $\mu_{i 1}$ of (13) can be derived as:

$$
\begin{aligned}
\mu_{i 1}= & \int_{\tilde{G}_{r}}\left\{1-\exp \left[-\frac{(1-\tau)\left(p_{i+1}+p_{\mathrm{c}}\right)}{\eta \tau p_{\mathrm{e}} \min \left(1, d^{-\alpha}\right)}+\frac{g \min \left(1, \tilde{d}^{-\alpha}\right)}{\min \left(1, d^{-\alpha}\right)}\right]\right\} \\
& \times \exp (-g) \mathrm{d} g,
\end{aligned}
$$

where the integral is taken over the interval of $\tilde{G}_{r}$ as shown in (13). Corresponding to whether $\min \left(1, \tilde{d}^{-\alpha}\right)$ equals $\min \left(1, d^{-\alpha}\right)$ or not, we can derive $\mu_{i 1}$ as follows. If $\min \left(1, \tilde{d}^{-\alpha}\right)=\min \left(1, d^{-\alpha}\right)$, the probability of $(15)$ is derived as:

$$
\begin{aligned}
\mu_{i 1}= & \exp \left[-\frac{(1-\tau)\left(p_{i}+p_{\mathrm{c}}\right)}{\eta \tau p_{\mathrm{e}} \min \left(1, \tilde{d}^{-\alpha}\right)}\right]-\exp \left[-\frac{(1-\tau)\left(p_{i+1}+p_{\mathrm{c}}\right)}{\eta \tau p_{\mathrm{e}} \min \left(1, \tilde{d}^{-\alpha}\right)}\right] \\
& -\frac{(1-\tau)\left(p_{i+1}-p_{i}\right)}{\eta \tau p_{\mathrm{e}} \min \left(1, \tilde{d}^{-\alpha}\right)} \exp \left[-\frac{(1-\tau)\left(p_{i+1}+p_{\mathrm{c}}\right)}{\eta \tau p_{\mathrm{e}} \min \left(1, d^{-\alpha}\right)}\right] .
\end{aligned}
$$

Otherwise, if $\min \left(1, \tilde{d}^{-\alpha}\right) \neq \min \left(1, d^{-\alpha}\right)$, the probability of (15) can be derived as:

$$
\begin{aligned}
\mu_{i 1}= & \exp \left[-\frac{(1-\tau)\left(p_{i}+p_{\mathrm{c}}\right)}{\eta \tau p_{\mathrm{e}} \min \left(1, \tilde{d}^{-\alpha}\right)}\right]-\exp \left[-\frac{(1-\tau)\left(p_{i+1}+p_{\mathrm{c}}\right)}{\eta \tau p_{\mathrm{e}} \min \left(1, \tilde{d}^{-\alpha}\right)}\right] \\
& -\exp \left[-\frac{(1-\tau)\left(p_{i+1}+p_{\mathrm{c}}\right)}{\eta \tau p_{\mathrm{e}} \min \left(1, d^{-\alpha}\right)}\right] \frac{\min \left(1, d^{-\alpha}\right)}{\min \left(1, d^{-\alpha}\right)-\min \left(1, \tilde{d}^{-\alpha}\right)} \\
& \times\left\{\exp \left[\frac{(1-\tau)\left(p_{i}+p_{\mathrm{c}}\right)}{\eta \tau p_{\mathrm{e}} \min \left(1, d^{-\alpha}\right)}-\frac{(1-\tau)\left(p_{i}+p_{\mathrm{c}}\right)}{\eta \tau p_{\mathrm{e}} \min \left(1, \tilde{d}^{-\alpha}\right)}\right]\right. \\
& \left.-\exp \left[\frac{(1-\tau)\left(p_{i+1}+p_{\mathrm{c}}\right)}{\eta \tau p_{\mathrm{e}} \min \left(1, d^{-\alpha}\right)}-\frac{(1-\tau)\left(p_{i+1}+p_{\mathrm{c}}\right)}{\eta \tau p_{\mathrm{e}} \min \left(1, \tilde{d}^{-\alpha}\right)}\right]\right\} .
\end{aligned}
$$


Proposition 2. The probability $\mu_{i 2}$ of (14) can be derived as:

$$
\begin{aligned}
\mu_{i 2}= & \left\{\exp \left[-\frac{(1-\tau)\left(p_{i}+p_{\mathrm{c}}\right)}{\eta \tau p_{\mathrm{e}} \min \left(1, d^{-\alpha}\right)}\right]-\exp \left[-\frac{(1-\tau)\left(p_{i+1}+p_{\mathrm{c}}\right)}{\eta \tau p_{\mathrm{e}} \min \left(1, d^{-\alpha}\right)}\right]\right\} \\
& \times \int_{\tilde{G}_{r}} \exp \left\{-\left[1-\frac{\min \left(1, \tilde{d}^{-\alpha}\right)}{\min \left(1, d^{-\alpha}\right)}\right] g\right\} \mathrm{d} g,
\end{aligned}
$$

where the integral is taken over the interval of $\tilde{G}_{r}$ as shown in (14). Corresponding to whether $\min \left(1, d^{-\alpha}\right)$ equals $\min \left(1, \tilde{d}^{-\alpha}\right)$ or not, we can derive $\mu_{i 2}$ as follows. If $\min \left(1, \tilde{d}^{-\alpha}\right)=\min \left(1, d^{-\alpha}\right)$, the probability of (18) can be derived as:

$$
\begin{aligned}
\mu_{i 2}= & \left\{\exp \left[-\frac{(1-\tau)\left(p_{i}+p_{\mathrm{c}}\right)}{\eta \tau p_{\mathrm{e}} \min \left(1, d^{-\alpha}\right)}\right]-\exp \left[-\frac{(1-\tau)\left(p_{i+1}+p_{\mathrm{c}}\right)}{\eta \tau p_{\mathrm{e}} \min \left(1, d^{-\alpha}\right)}\right]\right\} \\
& \times \frac{(1-\tau)\left(p_{i}+p_{\mathrm{c}}\right)}{\eta \tau p_{\mathrm{e}} \min \left(1, \tilde{d}^{-\alpha}\right)} .
\end{aligned}
$$

Otherwise, if $\min \left(1, \tilde{d}^{-\alpha}\right) \neq \min \left(1, d^{-\alpha}\right)$, the probability of (18) can be derived as:

$$
\begin{aligned}
\mu_{i 2}= & \frac{\min \left(1, d^{-\alpha}\right)}{\min \left(1, d^{-\alpha}\right)-\min \left(1, \tilde{d}^{-\alpha}\right)} \\
& \times\left\{1-\exp \left[\frac{(1-\tau)\left(p_{i}+p_{\mathrm{c}}\right)}{\eta \tau p_{\mathrm{e}} \min \left(1, d^{-\alpha}\right)}-\frac{(1-\tau)\left(p_{i}+p_{\mathrm{c}}\right)}{\eta \tau p_{\mathrm{e}} \min \left(1, \tilde{d}^{-\alpha}\right)}\right]\right\} \\
& \times\left\{\exp \left[-\frac{(1-\tau)\left(p_{i}+p_{\mathrm{c}}\right)}{\eta \tau p_{\mathrm{e}} \min \left(1, d^{-\alpha}\right)}\right]-\exp \left[-\frac{(1-\tau)\left(p_{i+1}+p_{\mathrm{c}}\right)}{\eta \tau p_{\mathrm{e}} \min \left(1, d^{-\alpha}\right)}\right]\right\} .
\end{aligned}
$$

After obtaining the two probabilities $\mu_{i 1}$ and $\mu_{i 2}$ in the above two propositions by averaging over the random channel fadings from the destination and the relay to the source, the probability of choosing a certain power level $p_{i}$ $(i \in\{1, \ldots, N-1\})$ can thus be obtained as $\mu_{i}=\mu_{i 1}+\mu_{i 2}$. Moreover, the probability of choosing the power zero that is the cooperative link being silent can be expressed as $\mu_{0}=\operatorname{Pr}\left\{\tilde{p}_{o}<p_{1}\right\}$, and it is derived as:

$$
\begin{aligned}
\mu_{0}= & \operatorname{Pr}\left\{\tilde{G}_{s} \min \left(1, d^{-\alpha}\right)<\frac{(1-\tau)\left(p_{1}+p_{\mathrm{c}}\right)}{\eta \tau p_{\mathrm{e}}}-\tilde{G}_{r} \min \left(1, \tilde{d}^{-\alpha}\right)\right\} \\
= & \operatorname{Pr}\left\{\tilde{G}_{s}<\frac{(1-\tau)\left(p_{1}+p_{\mathrm{c}}\right)}{\eta \tau p_{\mathrm{e}} \min \left(1, d^{-\alpha}\right)}-\frac{\tilde{G}_{r} \min \left(1, \tilde{d}^{-\alpha}\right)}{\min \left(1, d^{-\alpha}\right)},\right. \\
& \left.\tilde{G}_{r}<\frac{(1-\tau)\left(p_{1}+p_{\mathrm{c}}\right)}{\eta \tau p_{\mathrm{e}} \min \left(1, \tilde{d}^{-\alpha}\right)}\right\} .
\end{aligned}
$$

This probability can be derived in the following proposition.

Proposition 3. The probability of (21) can derived as:

$$
\begin{aligned}
\mu_{0}= & \int_{\tilde{G}_{r}}\left\{1-\exp \left[-\frac{(1-\tau)\left(p_{1}+p_{\mathrm{c}}\right)}{\eta \tau p_{\mathrm{e}} \min \left(1, d^{-\alpha}\right)}+\frac{g \min \left(1, \tilde{d}^{-\alpha}\right)}{\min \left(1, d^{-\alpha}\right)}\right]\right\} \\
& \times \exp (-g) \mathrm{d} g
\end{aligned}
$$

where the integral is taken over the interval of $\tilde{G}_{r}$ as shown in (21). Corresponding to whether $\min \left(1, d^{-\alpha}\right)$ equals $\min \left(1, \tilde{d}^{-\alpha}\right)$ or not, the probability $\mu_{0}$ is derived as follows. If $\min \left(1, d^{-\alpha}\right)=\min \left(1, \tilde{d}^{-\alpha}\right)$, we can derive the probability of (22) as:

$$
\begin{aligned}
\mu_{0}= & 1-\exp \left[-\frac{(1-\tau)\left(p_{1}+p_{\mathrm{c}}\right)}{\eta \tau p_{\mathrm{e}} \min \left(1, \tilde{d}^{-\alpha}\right)}\right] \\
& -\exp \left[-\frac{(1-\tau)\left(p_{1}+p_{\mathrm{c}}\right)}{\eta \tau p_{\mathrm{e}} \min \left(1, d^{-\alpha}\right)}\right] \frac{(1-\tau)\left(p_{1}+p_{\mathrm{c}}\right)}{\eta \tau p_{\mathrm{e}} \min \left(1, \tilde{d}^{-\alpha}\right)} .
\end{aligned}
$$

Otherwise, if $\min \left(1, d^{-\alpha}\right) \neq \min \left(1, \tilde{d}^{-\alpha}\right)$, we can derive the probability of (22) as:

$$
\begin{aligned}
\mu_{0}= & 1-\exp \left[-\frac{(1-\tau)\left(p_{1}+p_{\mathrm{c}}\right)}{\eta \tau p_{\mathrm{e}} \min \left(1, \tilde{d}^{-\alpha}\right)}\right] \\
& -\exp \left[-\frac{(1-\tau)\left(p_{1}+p_{\mathrm{c}}\right)}{\eta \tau p_{\mathrm{e}} \min \left(1, d^{-\alpha}\right)}\right] \frac{\min \left(1, d^{-\alpha}\right)}{\min \left(1, d^{-\alpha}\right)-\min \left(1, \tilde{d}^{-\alpha}\right)} \\
& \times\left\{1-\exp \left[\frac{(1-\tau)\left(p_{1}+p_{\mathrm{c}}\right)}{\eta \tau p_{\mathrm{e}} \min \left(1, d^{-\alpha}\right)}-\frac{(1-\tau)\left(p_{1}+p_{\mathrm{c}}\right)}{\eta \tau p_{\mathrm{e}} \min \left(1, \tilde{d}^{-\alpha}\right)}\right]\right\} .
\end{aligned}
$$

In the above proposition, we analyze the probability of the cooperative link keeping silent, that is, the probability of choosing the zero transmission power. Moreover, the probability of choosing the maximal power level $p_{N}$ can be expressed as $\mu_{N}=\operatorname{Pr}\left\{p_{N} \leq \tilde{p}_{o}\right\}$, and it is derived as:

$$
\begin{aligned}
\mu_{N}= & \operatorname{Pr}\left\{\tilde{G}_{s} \min \left(1, d^{-\alpha}\right) \geq \frac{(1-\tau)\left(p_{N}+p_{\mathrm{c}}\right)}{\eta \tau p_{\mathrm{e}}}-\tilde{G}_{r} \min \left(1, \tilde{d}^{-\alpha}\right)\right\} \\
= & \underbrace{\operatorname{Pr}\left\{\tilde{G}_{s} \geq \frac{(1-\tau)\left(p_{N}+p_{\mathrm{c}}\right)}{\eta \tau p_{\mathrm{e}} \min \left(1, d^{-\alpha}\right)}-\frac{\tilde{G}_{r} \min \left(1, \tilde{d}^{-\alpha}\right)}{\min \left(1, d^{-\alpha}\right)}, \tilde{G}_{r} \geq \frac{(1-\tau)\left(p_{N}+p_{\mathrm{c}}\right)}{\eta \tau p_{\mathrm{e}} \min \left(1, \tilde{d}^{-\alpha}\right)}\right\}}_{\mu_{N 1}} \\
& +\underbrace{\operatorname{Pr}\left\{\tilde{G}_{s} \geq \frac{(1-\tau)\left(p_{N}+p_{\mathrm{c}}\right)}{\eta \tau p_{\mathrm{e}} \min \left(1, d^{-\alpha}\right)}-\frac{\tilde{G}_{r} \min \left(1, \tilde{d}^{-\alpha}\right)}{\min \left(1, d^{-\alpha}\right)}, \tilde{G}_{r}<\frac{(1-\tau)\left(p_{N}+p_{\mathrm{c}}\right)}{\eta \tau p_{\mathrm{e}} \min \left(1, \tilde{d}^{-\alpha}\right)}\right\}}_{\mu_{N 2}},
\end{aligned}
$$


where the first probability can be derived as $\mu_{N 1}=$ $\exp \left[-\frac{(1-\tau)\left(p_{N}+p_{\mathrm{c}}\right)}{\eta \tau p_{\mathrm{e}} \min \left(1, \tilde{d}^{-\alpha}\right)}\right]$. The second probability of $(25)$ can be derived in the following proposition.

Proposition 4. The second probability $\mu_{N 2}$ of (25) can be derived as:

$$
\begin{aligned}
\mu_{N 2}= & \int_{\tilde{G}_{r}} \exp \left[-\frac{(1-\tau)\left(p_{N}+p_{\mathrm{c}}\right)}{\eta \tau p_{\mathrm{e}} \min \left(1, d^{-\alpha}\right)}+\frac{g \min \left(1, \tilde{d}^{-\alpha}\right)}{\min \left(1, d^{-\alpha}\right)}\right] \\
& \times \exp (-g) \mathrm{d} g
\end{aligned}
$$

where the integral is taken over the given interval of $\tilde{G}_{r}$ as shown in (25). Corresponding to whether $\min \left(1, d^{-\alpha}\right)$ equals $\min \left(1, \tilde{d}^{-\alpha}\right)$ or not, we can derive $\mu_{N 2}$ as follows. If $\min \left(1, \tilde{d}^{-\alpha}\right)=\min \left(1, d^{-\alpha}\right)$, the probability of (26) can be derived as:

$$
\mu_{N 2}=\exp \left[-\frac{(1-\tau)\left(p_{N}+p_{\mathrm{c}}\right)}{\eta \tau p_{\mathrm{e}} \min \left(1, d^{-\alpha}\right)}\right] \frac{(1-\tau)\left(p_{N}+p_{\mathrm{c}}\right)}{\eta \tau p_{\mathrm{e}} \min \left(1, \tilde{d}^{-\alpha}\right)}
$$

Otherwise, if $\min \left(1, \tilde{d}^{-\alpha}\right) \neq \min \left(1, d^{-\alpha}\right)$, the probability of (26) can be derived as:

$$
\begin{aligned}
\mu_{N 2}= & \exp \left[-\frac{(1-\tau)\left(p_{N}+p_{\mathrm{c}}\right)}{\eta \tau p_{\mathrm{e}} \min \left(1, d^{-\alpha}\right)}\right] \frac{\min \left(1, d^{-\alpha}\right)}{\min \left(1, d^{-\alpha}\right)-\min \left(1, \tilde{d}^{-\alpha}\right)} \\
& \times\left\{1-\exp \left[\frac{(1-\tau)\left(p_{N}+p_{\mathrm{c}}\right)}{\eta \tau p_{\mathrm{e}} \min \left(1, d^{-\alpha}\right)}-\frac{(1-\tau)\left(p_{N}+p_{\mathrm{c}}\right)}{\eta \tau p_{\mathrm{e}} \min \left(1, \tilde{d}^{-\alpha}\right)}\right]\right\} .
\end{aligned}
$$

Figure 3 shows the probability of choosing each power level with respect to the time allocation factor $\tau$. The system parameters are set the same as that adopted in Figure 2, and the relative distance between the relay and the source is set as $\tilde{d}=7.5 \mathrm{~m}$. The probability of choosing the zero/peak transmission power monotonically decreases/increases with the prolonging of the $\mathrm{EH}$ time. In each time block, the more time allocated for the $\mathrm{EH}$, the shorter time is retained for the data transmission, so it is more likely to choose a higher power level. For the other power levels, with the increase of the EH time fraction $\tau$, the probability of choosing each power level gets larger first and then turns smaller. This is because more energy is harvested with the increase of $\tau$, and it is more possible to choose the higher power level.

Figure 4 shows the probability of choosing a power level with respect to the EH time $\tau$ for different distances between the relay and the source. There are totally six power levels with $N=5$, and we focus on the second power level that is $\hat{p} / 5$. It can be seen that the source-relay distance can apparently impact the transmission power of the source. When the source-relay distance gets longer, i.e., the location parameter $\beta$ gets larger, the WET efficiency from both the relay and the destination becomes worse, and less amount of energy is harvested by the source. Consequently, it is more likely to choose the low power level and the maximal probability of choosing the given power level shifts to the right on the time line.

\section{Area throughput of the non-cooperative system} In each time block, after the EH in the first $\tau$ time fraction, the remaining $1-\tau$ time fraction is divided into two subperiods with equal length for the data transmission. In the first subperiod, the source transmits a data packet to the destination. If the data packet is correctly received by the destination, the source will transmit a new data packet in the second subperiod. Otherwise, if the data packet is erroneously received by the destination, the source will retransmit the same data packet in the second subperiod. Both the original and retransmitted data packets are maximal ratio combined (MRC) for the decoding. In this section, we will analyze the success probabilities of data transmission and retransmission, based on which the maximal area throughput of the non-cooperative system is investigated.

\subsection{Data success probability}

Since the EH amount varies for different sources due to the independent channel fading, the transmission power of each source is generally different. In the following, we will analyze the impacts of different transmission powers to the data success probability. We pick a typical link with the destination located at the origin, the performance of this particular link can reflect the whole network, and this assumption does not violate the distribution of other terminals according to the Slivnyak's theorem [22]. The signal-to-interference-plus-noise ratio (SINR) of the data transmission over the typical link is given as:

$$
\gamma_{o}=\frac{p_{o} G_{o} \min \left(1, d^{-\alpha}\right)}{\mathcal{I}_{o}+N_{0}}
$$

where $p_{o}$ is the transmission power of the typical source node, $G_{o}$ represents the channel power fading over the typical link for the data transmission, and $N_{0}$ is the power 


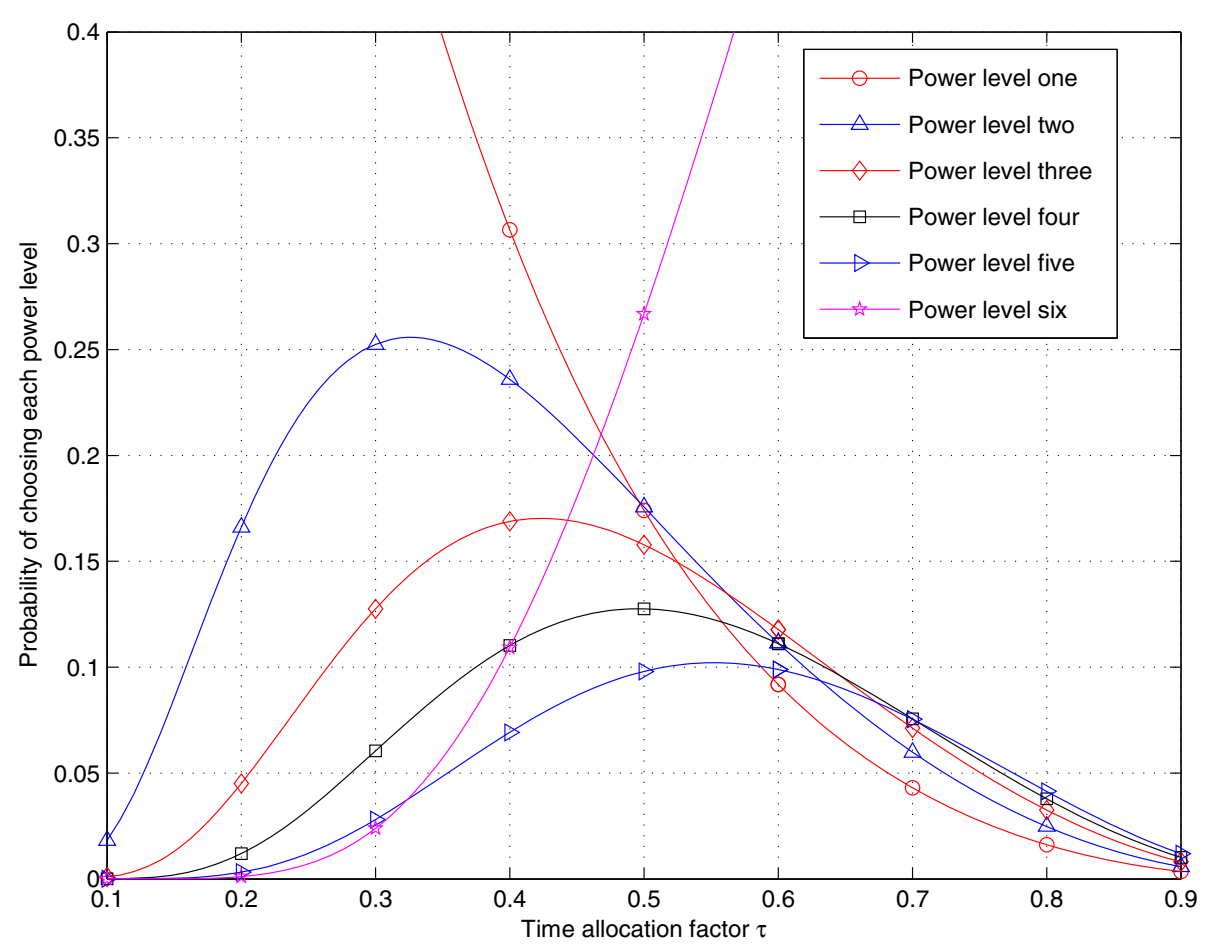

Figure 3 Probability of choosing each power level w.r.t. the time allocation factor $\tau$ for the cooperative WET.

of noise. Let $x_{o}$ denote the typical source node. The aggregate interference at the typical destination is modeled as:

$$
\mathcal{I}_{o}=\sum_{x \in \Pi_{\mathrm{s}} /\left\{x_{o}\right\}} p_{x} G_{x} \min \left(1, \ell_{x}^{-\alpha}\right)
$$

where all the active source nodes except the typical one contribute to the aggregate interference. The transmission power of the interferer $x \in \Pi_{\mathrm{s}} /\left\{x_{o}\right\}$ is denoted as $p_{x}$. The channel power fading and the distance between the interferer $x$ and the typical destination are denoted as $G_{x}$ and $\ell_{x}$, respectively.

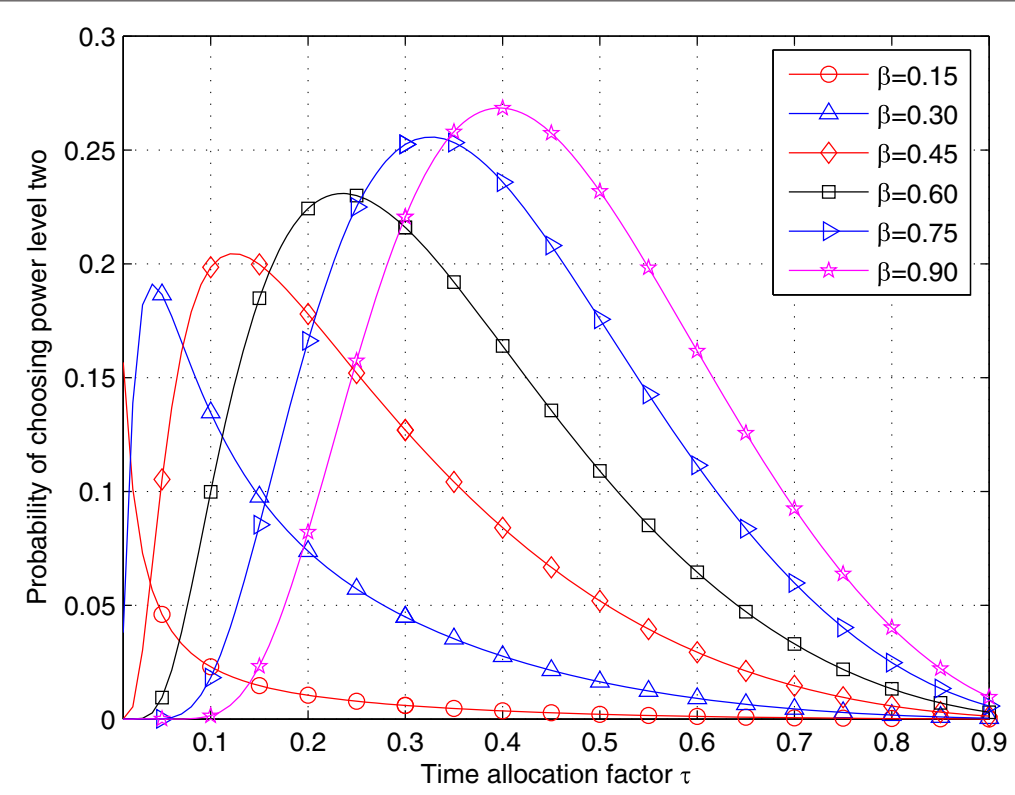

Figure 4 Probability of choosing the second power level w.r.t. the time allocation factor $\tau$ for the cooperative WET with different distance factors $\beta$. 
Let $\gamma_{t}=2^{\frac{R}{1-\beta}}-1$ denote the SINR threshold to judge whether the data transmission is successful or not, where $R$ is the fixed transmission rate of the source. The success probability of data transmission in the first subperiod is $P_{\text {suc1 }}^{\mathrm{n}}=\operatorname{Pr}\left\{\gamma_{o} \geq \gamma_{t}\right\}$, which can be derived as:

$$
\begin{aligned}
P_{\mathrm{suc} 1}^{\mathrm{n}}= & \operatorname{Pr}\left\{G_{o} \geq \frac{\gamma_{t}\left(\mathcal{I}_{o}+N_{0}\right)}{p_{o} \min \left(1, d^{-\alpha}\right)}\right\} \\
= & \mathbb{E}_{p_{o}, \mathcal{I}_{o}}\left\{\exp \left[-\frac{\gamma_{t}\left(\mathcal{I}_{o}+N_{0}\right)}{p_{o} \min \left(1, d^{-\alpha}\right)}\right]\right\} \\
= & \sum_{i=0}^{N} \delta_{i} \exp \left[-\frac{\gamma_{t} N_{0}}{p_{i} \min \left(1, d^{-\alpha}\right)}\right] \\
& \times \underbrace{\mathbb{E}_{\mathcal{I}_{o}}\left\{\exp \left[-\frac{\gamma_{t} \mathcal{I}_{o}}{p_{i} \min \left(1, d^{-\alpha}\right)}\right]\right\}}_{\mathcal{A}\left(p_{i}\right)},
\end{aligned}
$$

where we first take the expectation over the discrete transmission powers for the typical source by considering the independence of $p_{o}$ and $\mathcal{I}_{o}$. The parameter $\delta_{i}$ in (31) has been obtained in Equation 3. Given the transmission power of the typical source as $p_{i}$, the remaining expectation of (31) is taken over the aggregate interference, and it is derived as:

$$
\begin{aligned}
& \mathcal{A}\left(p_{i}\right) \\
& \stackrel{(a)}{=} \mathbb{E}_{\Pi_{\mathrm{s}}}\left\{\prod_{x \in \Pi_{\mathrm{s}}} \mathbb{E}_{G_{x}, p_{x}}\left[\exp \left(-\frac{\gamma_{t} p_{x} G_{x} \min \left(1, \ell_{x}^{-\alpha}\right)}{p_{i} \min \left(1, d^{-\alpha}\right)}\right)\right]\right\} \\
& \stackrel{(b)}{=} \exp \left\{-2 \pi \lambda_{\mathrm{s}} \int_{0}^{\infty}\left\{1-\mathbb{E}_{G_{x}, p_{x}}\left[\exp \left(-\frac{\gamma_{t} p_{x} G_{x} \min \left(1, \ell_{x}^{-\alpha}\right)}{p_{i} \min \left(1, d^{-\alpha}\right)}\right)\right]\right\} \ell \mathrm{d} \ell\right\} \\
& \stackrel{(c)}{=} \exp \left\{-2 \pi \lambda_{s} \sum_{j=0}^{N} \delta_{j} \int_{0}^{\infty}\left\{1-\mathbb{E}_{G_{x}}\left[\exp \left(-\frac{\gamma_{t} p_{j} G_{x} \min \left(1, \ell_{x}^{-\alpha}\right)}{p_{i} \min \left(1, d^{-\alpha}\right)}\right)\right]\right\} \ell \mathrm{d} \ell\right\} \\
& \stackrel{(d)}{=} \exp \left\{-2 \pi \lambda_{\mathrm{s}} \sum_{j=0}^{N} \delta_{j} \int_{0}^{\infty}\{\underbrace{\int_{0}^{\infty}\left[1-\exp \left(-\frac{\gamma_{t} p_{j} g \min \left(1, \ell^{-\alpha}\right)}{p_{i} \min \left(1, d^{-\alpha}\right)}\right)\right] \ell \mathrm{d} \ell}_{\mathcal{B}\left(p_{i}, p_{j}\right)}\} e^{-g} \mathrm{~d} g\right\},
\end{aligned}
$$

where $(a)$ is derived by separating the point process with the other random variables, $(b)$ is obtained according to the PGFL of PPP [22], (c) follows by taking the expectation over the discrete power levels, and $(d)$ is obtained by exchanging the integral orders of the channel fading and the distance. By taking the expectation over the random distance $\ell$ in the intervals $(0,1)$ and $(1, \infty)$, the inner integral of $(32)$ can be derived as:

$$
\begin{aligned}
\mathcal{B}\left(p_{i}, p_{j}\right)= & \frac{1}{2}\left[1-\exp \left(-\frac{\gamma_{t} p_{j} g}{p_{i} \min \left(1, d^{-\alpha}\right)}\right)\right] \\
& +\int_{1}^{\infty}\left[1-\exp \left(-\frac{\gamma_{t} p_{j} g \ell^{-\alpha}}{p_{i} \min \left(1, d^{-\alpha}\right)}\right)\right] \ell \mathrm{d} \ell .
\end{aligned}
$$

Substitute (33) into (32), after some mathematical manipulations according to the proof of Theorem 1 in [24], we can obtain:

$$
\begin{aligned}
\mathcal{A}\left(p_{i}\right)= & \prod_{j=0}^{N} \exp \left\{-\pi \lambda_{s} \delta_{j}\left[\frac{\gamma_{t} p_{j}}{p_{i} \min \left(1, d^{-\alpha}\right)}\right]^{\frac{2}{\alpha}}\right. \\
& \times\left[\frac{2 \pi / \alpha}{\sin (2 \pi / \alpha)}-\int_{0}^{\infty} g^{\frac{2}{\alpha}} \Gamma\left(1-\frac{2}{\alpha}, \frac{\gamma_{t} p_{j} g}{p_{i} \min \left(1, d^{-\alpha}\right)}\right)\right. \\
& \times \exp (-g) \mathrm{d} g]\},
\end{aligned}
$$

where $\Gamma(a, x)=\int_{x}^{\infty} e^{-t} t^{a-1} \mathrm{~d} t$ is the incomplete gamma function [25]. Substitute (34) into (31), we can obtain the success probability of source transmission in the first subperiod.

If the data transmission in the first subperiod is successful, the data transmission in the second subperiod is also deemed to be successful as the channel undergoes block fading and the interference is almost invariant. However, if the original data transmission fails, the retransmission is performed by the source with SINR $2 \gamma_{o}$ for the MRC detection at the destination. The success probability of data retransmission is expressed as $P_{\text {suc2 }}^{\mathrm{n}}=$ $\operatorname{Pr}\left\{\gamma_{o}<\gamma_{t}, 2 \gamma_{o} \geq \gamma_{t}\right\}$, and it can be derived as follows after some mathematical manipulations, i.e.:

$$
\begin{aligned}
P_{\text {suc2 }}^{\mathrm{n}}= & \operatorname{Pr}\left\{\frac{\gamma_{t}\left(\mathcal{I}_{o}+N_{0}\right)}{2 p_{o} \min \left(1, d^{-\alpha}\right)} \leq G_{o}<\frac{\gamma_{t}\left(\mathcal{I}_{o}+N_{0}\right)}{p_{o} \min \left(1, d^{-\alpha}\right)}\right\} \\
= & \mathbb{E}_{p_{o}, \mathcal{I}_{o}}\left\{\exp \left[-\frac{\gamma_{t}\left(\mathcal{I}_{o}+N_{0}\right)}{2 p_{o} \min \left(1, d^{-\alpha}\right)}\right]\right\} \\
& -\mathbb{E}_{p_{o}, \mathcal{I}_{o}}\left\{\exp \left[-\frac{\gamma_{t}\left(\mathcal{I}_{o}+N_{0}\right)}{p_{o} \min \left(1, d^{-\alpha}\right)}\right]\right\},
\end{aligned}
$$

where the second expectation term has been derived in (31), and the first expectation term can be similarly derived as (31) by replacing $p_{i}$ with $2 p_{i}$. 


\subsection{Achievable area throughput}

In the above subsection, we have derived the success probabilities of data transmission and retransmission for the non-cooperative wireless energy harvesting and data transmission system. The area throughput with unit $\mathrm{bits} / \mathrm{s} / \mathrm{Hz} / \mathrm{m}^{2}$ represents the effective data transmission rates per link multiplied by the average number of links in a unit area. Based on the above analysis, we define the achievable area throughput of the non-cooperative system as:

$$
\mathcal{T}_{\mathrm{n}}=\max _{\tau \in(0,1)} \lambda_{\mathrm{s}} R\left(P_{\mathrm{suc} 1}^{\mathrm{n}}+\frac{P_{\mathrm{suc} 2}^{\mathrm{n}}}{2}\right)
$$

where $R$ is the transmission rate and we have the relationship $\gamma_{t}=2^{\frac{R}{1-\tau}}-1$. The maximal area throughput of the ad hoc network can be obtained by searching over the time allocation factor $\tau$ in the interval $(0,1)$. The two success probabilities are dependent with $\tau$, and it is difficult to determine a closed-form solution for this parameter. Next, we will show the variation of the achievable area throughput with respect to the time allocation factor $\tau$.

Figure 5 shows the area throughput of the noncooperative system with respect to the time allocation factor $\tau$ for different transmission rates $R$. Without stating otherwise, the density of source nodes is set as $\lambda_{\mathrm{s}}=10^{-3}$ and the power of noise is set as $N_{0}=10^{-6} \mathrm{~W}$. It can be seen that the area throughput first increases and then decreases when the $\mathrm{EH}$ time $\tau$ gets longer from nearly 0 to nearly 1 . With the increase of the time fraction $\tau$, more energy can be harvested by the source, which is helpful to improve the data success probability, but less time is retained for the data transmission, which is harmful to enhance the area throughput. As a compromise, there exists an optimal time factor $\tau$ that can maximize the system area throughput. Moreover, when the transmission rate $R$ gets larger from 0.5 to $4.0 \mathrm{bits} / \mathrm{s} / \mathrm{Hz}$, the area throughput improves first and then deteriorates. In the lower regime of $R$, the increase of the transmission rate can beat against the adverse impacts of the success probability decrease, so the system performance becomes better. However, in the higher regime of $R$, the increase of the transmission rate cannot combat the severe degradation of the data success probability, so the system performance turns to be worse.

\section{Area throughput of the cooperative system}

In this section, we will analyze the data success probabilities, based on which the achievable area throughput is investigated for the cooperative WET and data relaying system. The energy and data transmission over each link is assisted by a fixed relay located on the line connecting the source and its destination. This assumption is approximate when the density of relay nodes is much higher and almost surely a relay can be found in a very small area at a certain location.

\subsection{Success probability of source transmission}

In each time block, using the energy harvested in the first $\tau$ time fraction, the cooperative data transmission is

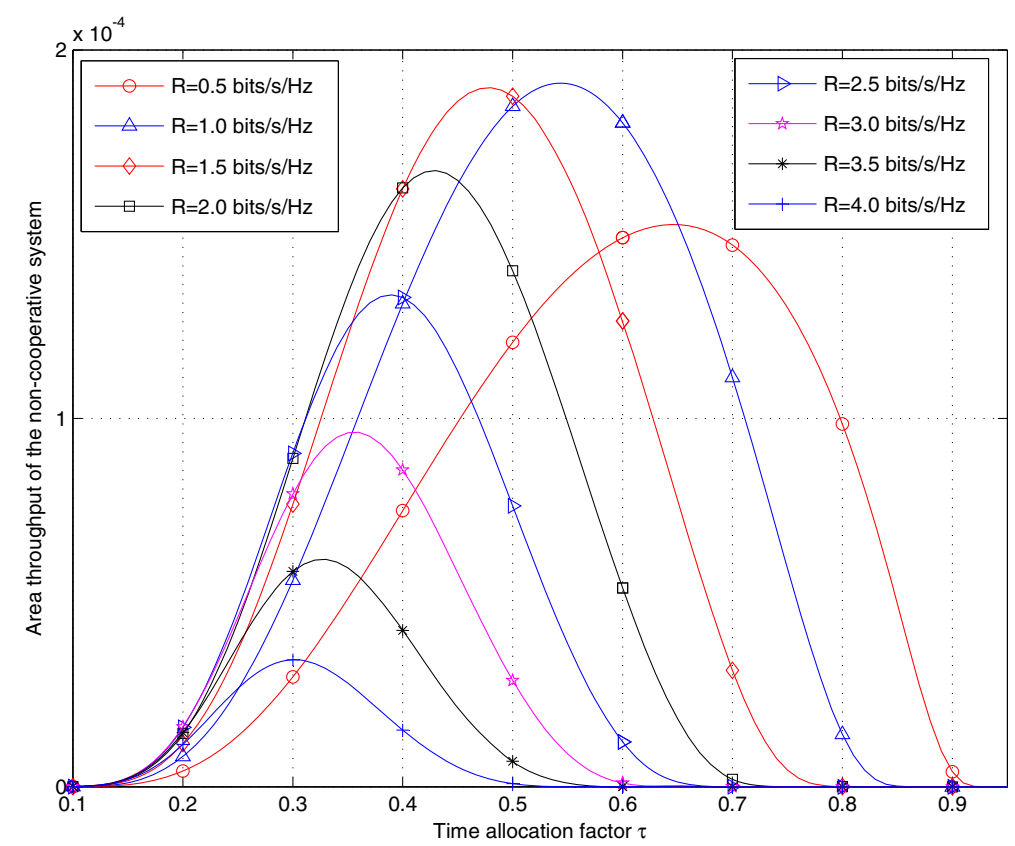

Figure 5 Area throughput of the non-cooperative system w.r.t. the time allocation factor $\tau$ for different transmission rates $R$. 
performed in the following $1-\tau$ time fraction, which is divided into two equal-length subperiods. In the first subperiod, the source broadcasts its data to both the relay and the destination. Due to the broadcast nature of the wireless channel, the source data can be overheard by both the destination and the relay at the same time. If the destination can successfully detect this data packet, the relay will keep silent and the source will transmit a new data packet in the second subperiod. Otherwise, if the destination erroneously receives the data, both the source and the relay will simultaneously forward the data in the second subperiod if the relay has correctly received the source data in the first subperiod. However, if the relay has no correct data copy, the source will retransmit the same data packet alone. The original and retransmitted data packets are combined by the destination for the joint decoding using the MRC technique.

The success probability of data transmission in the first subperiod is denoted as $P_{\text {suc } 1}^{\mathrm{c}}$, and it can be similarly derived as (31) by replacing $\delta_{i}$ and $\delta_{j}$ with $\mu_{i}$ and $\mu_{j}$, respectively. If the data transmission in the first subperiod is successful, the new source data transmission in the second subperiod may be unsuccessful. In the second subperiod, the interference power from other concurrent links is different from the first subperiod, as some relays of other links are active for the data retransmission with fixed power. The success probability of the data transmission in the second subperiod is denoted as $P_{\text {suc2 }}^{\mathrm{c}}$, and it is expressed as:

$$
P_{\text {suc2 }}^{\mathrm{c}}=\operatorname{Pr}\left\{\gamma_{o} \geq \gamma_{\mathrm{t}}, \tilde{\gamma}_{o} \geq \gamma_{\mathrm{t}}\right\}
$$

where $\gamma_{o}$ given by (29) represents the SINR of the typical destination in the first subperiod, and $\tilde{\gamma}_{o}$ represents the SINR of the typical destination in the second subperiod, and it is given as:

$$
\tilde{\gamma}_{o}=\frac{p_{o} G_{o} \min \left(1, d^{-\alpha}\right)}{\tilde{\mathcal{I}}_{o}+N_{0}}
$$

where the channel fading keeps invariant in the two successive subperiods, and the aggregate interference caused to the typical destination in the second subperiod is:

$$
\begin{aligned}
\tilde{\mathcal{I}}_{o}= & \sum_{x \in \Pi_{\mathrm{s}} /\left\{x_{o}\right\}} p_{x} G_{x} \min \left(1, \ell_{x}{ }^{-\alpha}\right) \\
& +\mathbf{1}\left(\gamma_{x}<\gamma_{\mathrm{t}}, \gamma_{x r} \geq \gamma_{\mathrm{t}}\right) p_{\mathrm{r}} G_{x r o} \min \left(1, \ell_{x}^{-\alpha}\right),
\end{aligned}
$$

where the indicator random variable $\mathbf{1}\left(\gamma_{x}<\gamma_{\mathrm{t}}, \gamma_{x r} \geq \gamma_{\mathrm{t}}\right)$ equals one if $\gamma_{x}<\gamma_{\mathrm{t}}$ and $\gamma_{x r} \geq \gamma_{\mathrm{t}}$, otherwise, it equals zero. In the expression (39), the SINR between a source node $x \in \Pi_{\mathrm{s}} /\left\{x_{o}\right\}$ and its destination is denoted as $\gamma_{x}$, the SINR between a source node $x$ and its relay is denoted as $\gamma_{x r}$, and the channel fading between the relay of source $x$ and the typical destination is denoted as $G_{x r o}$. Since the source nodes are sparsely distributed over the 2-D plane, the average distance between different source-destination pairs is far. Hence, it is appropriate to assume that the interfering source $x \in \Pi /\left\{x_{o}\right\}$ and its relay have the same distance $\ell_{x}$ towards the typical destination at the origin. Therefore, at the typical destination, the interference from both the source $x \in \Pi /\left\{x_{o}\right\}$ and its relay undergoes the same path-loss. For a particular interfering source $x$, the indicator random variable of (39) represents that the source erroneously transmits its data to the destination but its relay correctly receives the data in the first subperiod, so the relay will join in the retransmission with power $p_{\mathrm{r}}$ in the second subperiod.

Before analyzing the data success probability of source transmission in the second subperiod, we first study the proportion of relays active for the data retransmission in the second subperiod over the whole plane. Let $\gamma_{s r}$ denote the SINR between the typical source and the typical relay, it is given as:

$$
\gamma_{s r}=\frac{p_{o} G_{s r} \min \left(1, \tilde{d}^{-\alpha}\right)}{\mathcal{I}_{s r}+N_{0}},
$$

where $G_{s r}$ and $\tilde{d}$ represent the channel power fading and the distance between the source and the relay, respectively. The interference encountered at the typical relay in the first subperiod is modeled as:

$$
\mathcal{I}_{s r}=\sum_{x \in \Pi_{\mathrm{s}} /\left\{x_{o}\right\}} p_{x} G_{x r} \min \left(1, \ell_{x}{ }^{-\alpha}\right),
$$

where the distance between the interferer $x \in \Pi_{\mathrm{s}} /\left\{x_{o}\right\}$ and the relay is assumed to be the same with the distance between this interferer and the typical destination at the origin. The channel fading between an interferer $x \in \Pi_{\mathrm{s}} /\left\{x_{o}\right\}$ and the typical relay is denoted as $G_{x r}$. When $\gamma_{o}<\gamma_{\mathrm{t}}$ and $\gamma_{s r} \geq \gamma_{\mathrm{t}}$, the relay will be active for the data retransmission in the second subperiod. The probability is denoted as $\zeta=\operatorname{Pr}\left\{\gamma_{o}<\gamma_{\mathrm{t}}, \gamma_{s r} \geq \gamma_{\mathrm{t}}\right\}$, and it is calculated as:

$$
\begin{aligned}
\zeta & =\operatorname{Pr}\left\{G_{o}<\frac{\gamma_{\mathrm{t}}\left(\mathcal{I}_{o}+N_{0}\right)}{p_{o} \min \left(1, d^{-\alpha}\right)}, G_{s r} \geq \frac{\gamma_{\mathrm{t}}\left(\mathcal{I}_{s r}+N_{0}\right)}{p_{o} \min \left(1, \tilde{d}^{-\alpha}\right)}\right\} \\
& =\mathbb{E}\left\{\left[1-\exp \left(-\frac{\gamma_{\mathrm{t}}\left(\mathcal{I}_{o}+N_{0}\right)}{p_{o} \min \left(1, d^{-\alpha}\right)}\right)\right] \exp \left[-\frac{\gamma_{\mathrm{t}}\left(\mathcal{I}_{s r}+N_{0}\right)}{p_{o} \min \left(1, \tilde{d}^{-\alpha}\right)}\right]\right\} \\
& =\mathbb{E}\left\{\exp \left[-\frac{\gamma_{\mathrm{t}}\left(\mathcal{I}_{s r}+N_{0}\right)}{p_{o} \min \left(1, \tilde{d}^{-\alpha}\right)}\right]\right\}-\mathbb{E}\left\{\operatorname { e x p } \left[-\frac{\gamma_{\mathrm{t}}\left(\mathcal{I}_{o}+N_{0}\right)}{p_{o} \min \left(1, d^{-\alpha}\right)}\right.\right. \\
& \left.\left.-\frac{\gamma_{\mathrm{t}}\left(\mathcal{I}_{s r}+N_{0}\right)}{p_{o} \min \left(1, \tilde{d}^{-\alpha}\right)}\right]\right\},
\end{aligned}
$$


where the expectation is taken over the channel fading and the interference. The distance between any interferer and the typical relay is assumed to be the same as the distance between this interferer and the typical destination, so the first expectation of (42) can be approximately derived according to (31) by replacing $\delta_{i}$ and $d$ with $\mu_{i}$ and $\tilde{d}$, respectively. The other expectation of (42) can be derived as:

$$
\begin{aligned}
\mathbb{E}\left\{\exp \left[-\frac{\gamma_{t}\left(\mathcal{I}_{o}+N_{0}\right)}{p_{o} \min \left(1, d^{-\alpha}\right)}-\frac{\gamma_{t}\left(\mathcal{I}_{s r}+N_{0}\right)}{p_{o} \min \left(1, \tilde{d}^{-\alpha}\right)}\right]\right\} \\
=\sum_{i=0}^{N} \mu_{i} \mathbb{E}_{\mathcal{I}_{o}, \mathcal{I}_{s r}}\left\{\exp \left[-\frac{\gamma_{t}\left(\mathcal{I}_{o}+N_{0}\right)}{p_{i} \min \left(1, d^{-\alpha}\right)}-\frac{\gamma_{t}\left(\mathcal{I}_{s r}+N_{0}\right)}{p_{i} \min \left(1, \tilde{d}^{-\alpha}\right)}\right]\right\} \\
=\sum_{i=0}^{N} \mu_{i} \exp \left[-\frac{\gamma_{t} N_{0}}{p_{i} \min \left(1, d^{-\alpha}\right)}-\frac{\gamma_{t} N_{0}}{p_{i} \min \left(1, \tilde{d}^{-\alpha}\right)}\right] \\
\times \underbrace{\mathbb{E}_{\mathcal{I}_{o}, \mathcal{I}_{s r}}\left\{\exp \left[-\frac{\gamma_{t} \mathcal{I}_{o}}{p_{i} \min \left(1, d^{-\alpha}\right)}-\frac{\gamma_{t} \mathcal{I}_{s r}}{p_{i} \min \left(1, \tilde{d}^{-\alpha}\right)}\right]\right\}}_{\mathcal{D}\left(p_{i}\right)} .
\end{aligned}
$$

In the above derivation, we first take the expectation over the transmission power of the typical link, then the remaining expectation is taken over the interference. Given the transmission power of the typical source as $p_{i}$, the expectation term of (43) can be further calculated as:

$$
\begin{aligned}
\mathcal{D}\left(p_{i}\right)= & \mathbb{E}_{\Pi_{\mathrm{s}}}\left\{\prod _ { x \in \Pi _ { \mathrm { s } } } \mathbb { E } _ { G _ { x } , p _ { x } , G _ { x r } } \left\{\exp \left[-\frac{\gamma_{t} p_{x} G_{x} \min \left(1, \ell_{x}^{-\alpha}\right)}{p_{i} \min \left(1, d^{-\alpha}\right)}\right]\right.\right. \\
& \left.\left.\times \exp \left[-\frac{\gamma_{t} p_{x} G_{x r} \min \left(1, \ell_{x}^{-\alpha}\right)}{p_{i} \min \left(1, \tilde{d}^{-\alpha}\right)}\right]\right\}\right\} \\
= & \mathbb{E}_{\Pi_{\mathrm{s}}}\left\{\prod _ { x \in \Pi _ { \mathrm { s } } } \mathbb { E } _ { p _ { x } } \left[\frac{p_{i} \min \left(1, d^{-\alpha}\right)}{p_{i} \min \left(1, d^{-\alpha}\right)+\gamma_{t} p_{x} \min \left(1, \ell_{x}^{-\alpha}\right)}\right.\right. \\
& \left.\left.\times \frac{p_{i} \min \left(1, \tilde{d}^{-\alpha}\right)}{p_{i} \min \left(1, \tilde{d}^{-\alpha}\right)+\gamma_{t} p_{x} \min \left(1, \ell_{x}^{-\alpha}\right)}\right]\right\} \\
= & \prod_{j=0}^{N} \exp \left\{-\pi \lambda_{\mathrm{s}} \mu_{j}\left[1-\frac{p_{i} \min \left(1, d^{-\alpha}\right)}{p_{i} \min \left(1, d^{-\alpha}\right)+\gamma_{t} p_{j}}\right.\right. \\
& \left.\left.\times \frac{p_{i} \min \left(1, \tilde{d}^{-\alpha}\right)}{p_{i} \min \left(1, \tilde{d}^{-\alpha}\right)+\gamma_{t} p_{j}}+2 \mathcal{E}\left(p_{i}, p_{j}\right)\right]\right\}
\end{aligned}
$$

where we first take the expectation over the independent channel fading variables $G_{x}$ and $G_{x r}$, and then the expectation is taken over the discrete power $p_{x}$ and the point process according to the PGFL. The intermediate function of (44) is given as:

$$
\begin{aligned}
& \mathcal{E}\left(p_{i}, p_{j}\right) \\
& =\int_{1}^{\infty}\left\{1-\frac{p_{i}^{2} \min \left(1, d^{-\alpha}\right) \min \left(1, \tilde{d}^{-\alpha}\right)}{\left[p_{i} \min \left(1, d^{-\alpha}\right)+\gamma_{t} p_{j} \ell^{-\alpha}\right]\left[p_{i} \min \left(1, \tilde{d}^{-\alpha}\right)+\gamma_{t} p_{j} \ell^{-\alpha}\right]}\right\} \ell \mathrm{d} \ell .
\end{aligned}
$$

The above integral can be numerically calculated over finite range of $\ell$. By substituting (44) into (43), we can derive the second expectation term of (42). Therefore, the probability of the relay being active for the data retransmission in the second subperiod has been derived as (42).

Based on the above probability of relay being active in the second subperiod, we then analyze the success probability (37) of source transmission in the second subperiod as follows, i.e.:

$$
\begin{aligned}
P_{\text {suc } 2}^{\mathrm{c}} & =\operatorname{Pr}\left\{G_{o} \geq \frac{\gamma_{\mathrm{t}}\left(\tilde{\mathcal{I}}_{o}+N_{0}\right)}{p_{o} \min \left(1, d^{-\alpha}\right)}\right\}=\mathbb{E}\left\{\exp \left[-\frac{\gamma_{\mathrm{t}}\left(\tilde{\mathcal{I}}_{o}+N_{0}\right)}{p_{o} \min \left(1, d^{-\alpha}\right)}\right]\right\} \\
& =\sum_{i=0}^{N} \mu_{i} \exp \left[-\frac{\gamma_{\mathrm{t}} N_{0}}{p_{i} \min \left(1, d^{-\alpha}\right)}\right] \underbrace{\mathbb{E}\left\{\exp \left[-\frac{\gamma_{\mathrm{t}} \tilde{\mathcal{I}}_{o}}{p_{i} \min \left(1, d^{-\alpha}\right)}\right]\right\}}_{\mathcal{F}\left(p_{i}\right)},
\end{aligned}
$$

where the expectation over the discrete power level is first taken and the expectation over the interference can be derived as:

$$
\begin{aligned}
\mathcal{F}\left(p_{i}\right) \stackrel{(a)}{=} & \mathbb{E}\left\{\prod_{x \in \Pi_{\mathrm{s}}} \exp \left[-\frac{\gamma_{\mathrm{t}} \min \left(1, \ell_{x}^{-\alpha}\right)\left[p_{x} G_{x}+\mathbf{1}\left(\gamma_{x}<\gamma_{\mathrm{t}}, \gamma_{x r} \geq \gamma_{\mathrm{t}}\right) p_{\mathrm{r}} G_{x r o}\right]}{p_{i} \min \left(1, d^{-\alpha}\right)}\right]\right\} \\
\stackrel{(b)}{\approx} & \mathbb{E}_{\Pi_{\mathrm{s}}}\left\{\prod _ { x \in \Pi _ { \mathrm { s } } } \mathbb { E } _ { p _ { x } , G _ { x } , G _ { x x o } } \left\{(1-\zeta) \exp \left[-\frac{\gamma_{\mathrm{t}} p_{x} G_{x} \min \left(1, \ell_{x}^{-\alpha}\right)}{p_{i} \min \left(1, d^{-\alpha}\right)}\right]\right.\right. \\
& \left.\left.+\zeta \exp \left[-\frac{\gamma_{\mathrm{t}} \min \left(1, \ell_{x}^{-\alpha}\right)\left(p_{x} G_{x}+p_{\mathrm{r}} G_{x r o}\right)}{p_{i} \min \left(1, d^{-\alpha}\right)}\right]\right\}\right\} \\
& \stackrel{(c)}{=} \mathbb{E}_{\Pi_{\mathrm{s}}}\left\{\prod _ { x \in \Pi _ { \mathrm { s } } } \mathbb { E } _ { p _ { x } } \left\{\frac{p_{i} \min \left(1, d^{-\alpha}\right)}{p_{i} \min \left(1, d^{-\alpha}\right)+\gamma_{\mathrm{t}} p_{x} \min \left(1, \ell_{x}-\alpha\right)}\right.\right. \\
& \left.\left.\times\left[(1-\zeta)+\frac{\zeta p_{i} \min \left(1, d^{-\alpha}\right)}{p_{i} \min \left(1, d^{-\alpha}\right)+\gamma_{\mathrm{t}} p_{\mathrm{r}} \min \left(1, \ell_{x}^{-\alpha}\right)}\right]\right\}\right\} \\
& \stackrel{(d)}{=} \prod_{j=0}^{N} \exp \left\{-\pi \lambda_{\mathrm{s}} \mu_{j}\left\{1-\frac{p_{i} \min \left(1, d^{-\alpha}\right)}{p_{i} \min \left(1, d^{-\alpha}\right)+\gamma_{\mathrm{t}} p_{j}}\right.\right. \\
& \left.\left.\times\left[(1-\zeta)+\frac{\zeta p_{i} \min \left(1, d^{-\alpha}\right)}{p_{i} \min \left(1, d^{-\alpha}\right)+\gamma_{\mathrm{t}} p_{\mathrm{r}}}\right]+2 \mathcal{G}\left(p_{i}, p_{j}\right)\right\}\right\},
\end{aligned}
$$

where $(a)$ is obtained by substituting the interference $\tilde{\mathcal{I}}_{o}$ given by (39), the approximation of $(b)$ is derived by assuming the indicator random variable is independent with other variables and each relay is active or not is independent with other users, $(c)$ is derived by taking the expectations over the two independent channel fading variables $G_{x}$ and $G_{x r o}$, and $(d)$ is obtained by taking the 
expectation over the discrete power $p_{x}$ and over the point process according to the PGFL. The intermediate function in (47) is defined as:

$$
\begin{aligned}
\mathcal{G}\left(p_{i}, p_{j}\right)= & \int_{1}^{\infty}\left\{1-\frac{p_{i} \min \left(1, d^{-\alpha}\right)}{p_{i} \min \left(1, d^{-\alpha}\right)+\gamma_{\mathrm{t}} p_{j} \ell^{-\alpha}}\right. \\
& \left.\times\left[(1-\zeta)+\frac{\zeta p_{i} \min \left(1, d^{-\alpha}\right)}{p_{i} \min \left(1, d^{-\alpha}\right)+\gamma_{\mathrm{t}} p_{\mathrm{r}} \ell^{-\alpha}}\right]\right\} \ell \mathrm{d} \ell .
\end{aligned}
$$

The intermediate function $\mathcal{G}\left(p_{i}, p_{j}\right)$ with one-dimensional integral can be numerically calculated. By substituting (47) into (46), the approximate success probability of source transmitting in the second subperiod is obtained, where the probability of the relay being active is included in the derivation.

\subsection{Success probability of source retransmission}

If the source data transmission in the first subperiod is unsuccessful, the retransmission will be performed in the second subperiod. When the relay does not correctly receive the source data in the first subperiod, the data retransmission is performed by the source, and the success probability of this event is denoted as $P_{\text {suc3 }}^{\mathrm{c}}$, i.e.:

$$
P_{\text {suc3 }}^{\mathrm{c}}=\operatorname{Pr}\left\{\gamma_{o}<\gamma_{t}, \gamma_{s r}<\gamma_{t}, \gamma_{o}+\tilde{\gamma}_{o} \geq \gamma_{t}\right\},
$$

where $\gamma_{o}<\gamma_{t}$ represents the data transmission in the first subperiod fails, $\gamma_{s r}<\gamma_{t}$ represents that the relay does not correctly receive the source data, and $\gamma_{o}+\tilde{\gamma}_{o} \geq \gamma_{t}$ represents that the data retransmission with MRC decoding is successful at the destination. The SINR between source and destination is $\gamma_{o}$ given in (29), the SINR between source and relay is $\gamma_{s r}$ given in (40), the SINR of the source retransmission is $\tilde{\gamma}_{o}$ given in (38). In the derivation, the distance between any interferer and the relay is assumed to be the same as that between the interferer and the typical destination. This assumption is appropriate when the density of the source links is small and the average distance between any two links is far.

By substituting the related SINR values into the probability expression, we can calculate the success probability of source retransmission as:

$$
\begin{aligned}
P_{\text {suc3 }}^{\mathrm{c}}= & \operatorname{Pr}\left\{\frac{p_{o} G_{o} \min \left(1, d^{-\alpha}\right)}{\mathcal{I}_{o}+N_{0}}<\gamma_{t}, \frac{p_{o} G_{s r} \min \left(1, \tilde{d}^{-\alpha}\right)}{\mathcal{I}_{s r}+N_{0}}<\gamma_{t},\right. \\
& \left.\frac{p_{o} G_{o} \min \left(1, d^{-\alpha}\right)}{\mathcal{I}_{o}+N_{0}}+\frac{p_{o} G_{o} \min \left(1, d^{-\alpha}\right)}{\tilde{\mathcal{I}}_{o}+N_{0}} \geq \gamma_{\mathrm{t}}\right\} .
\end{aligned}
$$

The probability of (50) is not easy to analyze. Instead, we try to derive two approximate results in the following analysis.
By omitting the interference from the relays, i.e., replacing $\tilde{\mathcal{I}}_{o}$ with $\mathcal{I}_{o}$, we can obtain an upper bound of the approximate success probability $P_{\text {suc3 }}^{\mathrm{c}}$. The success probability upper bound is denoted as $\hat{P}_{\text {suc3 }}^{\mathrm{c}}$, and it is derived as:

$$
\begin{aligned}
\hat{P}_{\text {suc } 3}^{\mathrm{c}}= & \operatorname{Pr}\left\{\frac{\gamma_{t}\left(\mathcal{I}_{o}+N_{0}\right)}{2 p_{o} \min \left(1, d^{-\alpha}\right)} \leq G_{o}<\frac{\gamma_{t}\left(\mathcal{I}_{o}+N_{0}\right)}{p_{o} \min \left(1, d^{-\alpha}\right)}, G_{s r}<\frac{\gamma_{t}\left(\mathcal{I}_{s r}+N_{0}\right)}{p_{o} \min \left(1, \tilde{d}^{-\alpha}\right)}\right\} \\
= & \mathbb{E}_{p_{o}, \mathcal{I}_{o}}\left\{\exp \left[-\frac{\gamma_{t}\left(\mathcal{I}_{o}+N_{0}\right)}{2 p_{o} \min \left(1, d^{-\alpha}\right)}\right]\right\}-\mathbb{E}_{p_{o}, \mathcal{I}_{o}}\left\{\exp \left[-\frac{\gamma_{t}\left(\mathcal{I}_{o}+N_{0}\right)}{p_{o} \min \left(1, d^{-\alpha}\right)}\right]\right\} \\
& +\mathbb{E}_{p_{o}, \mathcal{I}_{o}, \mathcal{I}_{s r}}\left\{\exp \left[-\frac{\gamma_{t}\left(\mathcal{I}_{o}+N_{0}\right)}{p_{o} \min \left(1, d^{-\alpha}\right)}-\frac{\gamma_{t}\left(\mathcal{I}_{s r}+N_{0}\right)}{p_{o} \min \left(1, \tilde{d}^{-\alpha}\right)}\right]\right\} \\
& -\mathbb{E}_{p_{o}, \mathcal{I}_{o}, \mathcal{I}_{s r}}\left\{\exp \left[-\frac{\gamma_{t}\left(\mathcal{I}_{o}+N_{0}\right)}{2 p_{o} \min \left(1, d^{-\alpha}\right)}-\frac{\gamma_{t}\left(\mathcal{I}_{s r}+N_{0}\right)}{p_{o} \min \left(1, \tilde{d}^{-\alpha}\right)}\right]\right\},
\end{aligned}
$$

where the first two expectations can be similarly derived according to (35) by replacing $\delta_{i}$ with $\mu_{i}$. There are two other similar expectations, and one expectation has been derived in (43). Similarly to the derivation of (43), the last expectation of (51) can be obtained by replacing $\min \left(1, d^{-\alpha}\right)$ with $2 \min \left(1, d^{-\alpha}\right)$.

In the MRC detection process, if we assume that the original data transmission is also interfered by the active relay nodes, i.e., by replacing $\mathcal{I}_{o}$ with $\tilde{\mathcal{I}}_{o}$ in the last term of (50), we can derive the lower bound of the approximate success probability. Denoted as $\check{P}_{\text {suc3 }}^{\mathrm{c}}$, the success probability lower bound is derived as:

$$
\begin{aligned}
\breve{P}_{\text {suc } 3}^{\mathrm{c}}= & \operatorname{Pr}\left\{\frac{\gamma_{\mathrm{t}}\left(\tilde{\mathcal{I}}_{o}+N_{0}\right)}{2 p_{o} \min \left(1, d^{-\alpha}\right)} \leq G_{o}<\frac{\gamma_{\mathrm{t}}\left(\mathcal{I}_{o}+N_{0}\right)}{p_{o} \min \left(1, d^{-\alpha}\right)}, G_{s r}<\frac{\gamma_{\mathrm{t}}\left(\mathcal{I}_{s r}+N_{0}\right)}{p_{o} \min \left(1, \tilde{d}^{-\alpha}\right)}\right\} \\
= & \mathbb{E}\left\{\exp \left[-\frac{\gamma_{\mathrm{t}}\left(\tilde{\mathcal{I}}_{o}+N_{0}\right)}{2 p_{o} \min \left(1, d^{-\alpha}\right)}\right]\right\}-\mathbb{E}\left\{\exp \left[-\frac{\gamma_{\mathrm{t}}\left(\mathcal{I}_{o}+N_{0}\right)}{p_{o} \min \left(1, d^{-\alpha}\right)}\right]\right\} \\
& +\mathbb{E}\left\{\exp \left[-\frac{\gamma_{\mathrm{t}}\left(\mathcal{I}_{o}+N_{0}\right)}{p_{o} \min \left(1, d^{-\alpha}\right)}-\frac{\gamma_{\mathrm{t}}\left(\mathcal{I}_{s r}+N_{0}\right)}{p_{o} \min \left(1, \tilde{d}^{-\alpha}\right)}\right]\right\} \\
& -\mathbb{E}\left\{\exp \left[-\frac{\gamma_{\mathrm{t}}\left(\tilde{\mathcal{I}}_{o}+N_{0}\right)}{2 p_{o} \min \left(1, d^{-\alpha}\right)}-\frac{\gamma_{\mathrm{t}}\left(\mathcal{I}_{s r}+N_{0}\right)}{p_{o} \min \left(1, \tilde{d}^{-\alpha}\right)}\right]\right\},
\end{aligned}
$$

where the first expectation can be derived according to (46) by replacing $p_{o}$ with $2 p_{o}$, the second expectation can be derived as (31) by replacing $\delta_{i}$ with $\mu_{i}$, the third expectation has been derived in (43). The last expectation of (52) is derived as:

$$
\begin{aligned}
& \mathbb{E}\left\{\exp \left[-\frac{\gamma_{\mathrm{t}}\left(\tilde{\mathcal{I}}_{o}+N_{0}\right)}{2 p_{o} \min \left(1, d^{-\alpha}\right)}-\frac{\gamma_{\mathrm{t}}\left(\mathcal{I}_{s r}+N_{0}\right)}{p_{o} \min \left(1, \tilde{d}^{-\alpha}\right)}\right]\right\} \\
& =\sum_{i=0}^{N} \mu_{i} \exp \left[-\frac{\gamma_{\mathrm{t}} N_{0}}{2 p_{i} \min \left(1, d^{-\alpha}\right)}\right] \exp \left[-\frac{\gamma_{\mathrm{t}} N_{0}}{p_{i} \min \left(1, \tilde{d}^{-\alpha}\right)}\right] \\
& \times \underbrace{\mathbb{E}\left\{\exp \left[-\frac{\gamma_{\mathrm{t}} \tilde{\mathcal{I}}_{o}}{2 p_{i} \min \left(1, d^{-\alpha}\right)}-\frac{\gamma_{\mathrm{t}} \mathcal{I}_{s r}}{p_{i} \min \left(1, \tilde{d}^{-\alpha}\right)}\right]\right\}}_{\mathcal{H}\left(p_{i}\right)},
\end{aligned}
$$


where the expectation over the transmission power of the typical source is taken and the remaining expectation is taken over the random interference. Let $\mathcal{R}$ denote the event that a certain relay is active for the data retransmission. Given the power level of the typical source as $p_{i}$, we can calculate the expectation term of (53) as:

$$
\begin{aligned}
& \mathcal{H}\left(p_{i}\right) \stackrel{(a)}{=} \mathbb{E}_{\Pi_{\mathrm{s}}}\left\{\prod _ { x \in \Pi _ { \mathrm { s } } } \mathbb { E } _ { G _ { x } , G _ { x r } , G _ { x r } , \mathcal { R } } \left\{\exp \left[-\frac{\gamma_{t} p_{x} G_{x r} \min \left(1, \ell_{x}^{-\alpha}\right)}{p_{i} \min \left(1, \tilde{d}^{-\alpha}\right)}\right]\right.\right. \\
& \left.\left.\times \exp \left[-\frac{\gamma_{\mathrm{t}}\left[p_{x} G_{x} \min \left(1, \ell_{x}^{-\alpha}\right)+\mathbf{1}\left(\gamma_{x}<\gamma_{\mathrm{t}}, \gamma_{x r} \geq \gamma_{\mathrm{t}}\right) p_{\mathrm{r}} G_{x r_{0}} \min \left(1, \ell_{x}^{-\alpha}\right)\right]}{2 p_{i} \min \left(1, d^{-\alpha}\right)}\right]\right\}\right\} \\
& \stackrel{(b)}{\approx} \mathbb{E}_{\Pi_{\mathrm{s}}}\left\{\prod _ { x \in \Pi _ { \mathrm { s } } } \mathbb { E } _ { p _ { x } , G _ { x } , G _ { x r } , G _ { x r o } } \left\{(1-\zeta) \exp \left[-\frac{\gamma_{\mathrm{t}} p_{x} G_{x r} \min \left(1, \ell_{x}^{-\alpha}\right)}{p_{i} \min \left(1, \tilde{d}^{-\alpha}\right)}\right]\right.\right. \\
& \times \exp \left[-\frac{\gamma_{\mathrm{t}} p_{x} G_{x} \min \left(1, \ell_{x}^{-\alpha}\right)}{2 p_{i} \min \left(1, d^{-\alpha}\right)}\right]+\zeta \exp \left[-\frac{\gamma_{\mathrm{t}} p_{x} G_{x r} \min \left(1, \ell_{x}^{-\alpha}\right)}{p_{i} \min \left(1, \tilde{d}^{-\alpha}\right)}\right] \\
& \left.\left.\times \exp \left[-\frac{\gamma_{\mathrm{t}}\left[p_{x} G_{x} \min \left(1, \ell_{x}^{-\alpha}\right)+p_{\mathrm{r}} G_{x r o} \min \left(1, \ell_{x}^{-\alpha}\right)\right]}{2 p_{i} \min \left(1, d^{-\alpha}\right)}\right]\right\}\right\} \\
& \stackrel{(c)}{=} \mathbb{E}_{\Pi_{\mathrm{s}}}\left\{\prod _ { x \in \Pi _ { \mathrm { s } } } \mathbb { E } _ { p _ { x } } \left\{\left[(1-\zeta)+\frac{2 \zeta p_{i} \min \left(1, d^{-\alpha}\right)}{2 p_{i} \min \left(1, d^{-\alpha}\right)+\gamma_{\mathrm{t}} p_{\mathrm{r}} \min \left(1, \ell_{x}-\alpha\right)}\right]\right.\right. \\
& \left.\left.\times \frac{2 p_{i}^{2} \min \left(1, \tilde{d}^{-\alpha}\right) \min \left(1, d^{-\alpha}\right)}{\left[p_{i} \min \left(1, \tilde{d}^{-\alpha}\right)+\gamma_{\mathrm{t}} p_{x} \min \left(1, \ell_{x}^{-\alpha}\right)\right]\left[2 p_{i} \min \left(1, d^{-\alpha}\right)+\gamma_{\mathrm{t}} p_{x} \min \left(1, \ell_{x}^{-\alpha}\right)\right]}\right\}\right\}
\end{aligned}
$$

where the approximate value is derived in $(b)$ by taking over the event $\mathcal{R}$. After taking the expectations over the independent channel fading random variables $G_{x}, G_{x r}$, and $G_{x r o}$, we can derive the result of $(c)$. After taking the expectation over the discrete transmission power $p_{x}$ and using the PGFL, we can further derive the following result, i.e.:

$$
\begin{aligned}
\mathcal{H}\left(p_{i}\right)= & \prod_{j=0}^{N} \exp \left\{-\pi \lambda_{\mathrm{s}} \mu_{j}\left\{1-\left[(1-\zeta)+\frac{2 \zeta p_{i} \min \left(1, d^{-\alpha}\right)}{2 p_{i} \min \left(1, d^{-\alpha}\right)+\gamma_{\mathrm{t}} p_{\mathrm{r}}}\right]\right.\right. \\
& \times \frac{2 p_{i}^{2} \min \left(1, \tilde{d}^{-\alpha}\right) \min \left(1, d^{-\alpha}\right)}{\left[p_{i} \min \left(1, \tilde{d}^{-\alpha}\right)+\gamma_{\mathrm{t}} p_{j}\right]\left[2 p_{i} \min \left(1, d^{-\alpha}\right)+\gamma_{\mathrm{t}} p_{j}\right]} \\
& \left.\left.+2 \mathcal{X}\left(p_{i}, p_{j}\right)\right\}\right\},
\end{aligned}
$$

where the intermediate function is defined as:

$$
\begin{aligned}
\mathcal{X}\left(p_{i}, p_{j}\right)= & \int_{1}^{\infty}\left\{1-\left[(1-\zeta)+\frac{2 \zeta p_{i} \min \left(1, d^{-\alpha}\right)}{2 p_{i} \min \left(1, d^{-\alpha}\right)+\gamma_{\mathrm{t}} p_{\mathrm{r}} \ell^{-\alpha}}\right]\right. \\
& \left.\times \frac{2 p_{i}^{2} \min \left(1, \tilde{d}^{-\alpha}\right) \min \left(1, d^{-\alpha}\right)}{\left[p_{i} \min \left(1, \tilde{d}^{-\alpha}\right)+\gamma_{\mathrm{t}} p_{j} \ell^{-\alpha}\right]\left[2 p_{i} \min \left(1, d^{-\alpha}\right)+\gamma_{\mathrm{t}} p_{j} \ell^{-\alpha}\right]}\right\} \ell \mathrm{d} \ell .
\end{aligned}
$$

The one-dimensional integral of $\mathcal{X}\left(p_{i}, p_{j}\right)$ can be numerically calculated. By substituting (55) into (53), we can obtain the last expectation of (52), so the result of the lower success probability $\breve{P}_{\text {suc3 }}^{\mathrm{c}}$ can be derived finally.

\subsection{Success probability of relay retransmission}

In the previous subsections, we have derived the success probability of source transmitting in the second subperiod when the original transmission towards the destination is successful in the first subperiod. Also, the success probability of source retransmitting is derived when the original transmission is unsuccessful and the relay has no correct data copy. In this subsection, we will analyze the success probability of the relay retransmitting when the original transmission fails in the first subperiod. Denoted as $P_{\text {suc4, }}^{\mathrm{c}}$, the success probability can be expressed as:

$$
P_{\text {suc4 }}^{\mathrm{c}}=\operatorname{Pr}\left\{\gamma_{o}<\gamma_{t}, \gamma_{s r} \geq \gamma_{t}, \gamma_{o}+\gamma_{r d} \geq \gamma_{t}\right\}
$$

where $\gamma_{o}<\gamma_{t}$ represents that the original data transmission fails in the first subperiod, $\gamma_{s r} \geq \gamma_{t}$ represents that the relay correctly receives the source data in the first subperiod, and $\gamma_{o}+\gamma_{r d} \geq \gamma_{t}$ represents that the MRC decoding with retransmission performed by the relay is successful. The SINR between the relay and the destination is denoted as $\gamma_{r d}$, which is given as:

$$
\gamma_{r d}=\frac{p_{\mathrm{r}} G_{r d} \min \left(1, \hat{d}^{-\alpha}\right)}{\tilde{\mathcal{I}}_{o}+N_{0}}
$$

where $\hat{d}=(1-\beta) d$ is the distance between the relay and the destination, and $p_{r}$ is the data transmission power of the relay node. The interference accumulated at the typical destination in the second subperiod is denoted as $\tilde{\mathcal{I}}_{o}$, and it is given in (39). Substituting the related terms into (57) and after some mathematical manipulations, we can obtain:

$$
\begin{aligned}
P_{\mathrm{suc} 4}^{\mathrm{c}}= & \operatorname{Pr}\left\{G_{o}<\frac{\gamma_{\mathrm{t}}\left(\mathcal{I}_{o}+N_{0}\right)}{p_{o} \min \left(1, d^{-\alpha}\right)}, G_{s r} \geq \frac{\gamma_{\mathrm{t}}\left(\mathcal{I}_{s r}+N_{0}\right)}{p_{o} \min \left(1, \tilde{d}^{-\alpha}\right)},\right. \\
& \left.\frac{p_{o} G_{o} \min \left(1, d^{-\alpha}\right)}{\mathcal{I}_{o}+N_{0}}+\frac{p_{\mathrm{r}} G_{r d} \min \left(1, \hat{d}^{-\alpha}\right)}{\tilde{\mathcal{I}}_{o}+N_{0}} \geq \gamma_{\mathrm{t}}\right\} .
\end{aligned}
$$

It is difficult to derive the closed-form solution for this probability, instead we will derive the upper and lower approximations similarly to the case of source retransmitting. Through omitting the interference from the relays, i.e., replacing $\tilde{\mathcal{I}}_{o}$ with $\mathcal{I}_{o}$ in (59), we can derive an upper 
bound of the approximate success probability, which is denoted as $\hat{P}_{\text {suc4 }}^{\mathrm{c}}$ and given by:

$$
\begin{aligned}
\hat{P}_{\text {suc } 4}^{\mathrm{c}} & \\
= & \mathbb{E}\left\{\frac{p_{\mathrm{r}} \min \left(1, \hat{d}^{-\alpha}\right)}{p_{\mathrm{r}} \min \left(1, \hat{d}^{-\alpha}\right)-p_{o} \min \left(1, d^{-\alpha}\right)} \exp \left[-\frac{\gamma_{\mathrm{t}}\left(\mathcal{I}_{s r}+N_{0}\right)}{p_{o} \min \left(1, \tilde{d}^{-\alpha}\right)}\right]\right. \\
& \left.\times \exp \left[-\frac{\gamma_{\mathrm{t}}\left(\mathcal{I}_{o}+N_{0}\right)}{p_{\mathrm{r}} \min \left(1, \hat{d}^{-\alpha}\right)}\right]\left\{1-\exp \left[\frac{\gamma_{\mathrm{t}}\left(\mathcal{I}_{o}+N_{0}\right)}{p_{\mathrm{r}} \min \left(1, \hat{d}^{-\alpha}\right)}-\frac{\gamma_{\mathrm{t}}\left(\mathcal{I}_{o}+N_{0}\right)}{p_{o} \min \left(1, d^{-\alpha}\right)}\right]\right\}\right\} \\
= & \sum_{i=0}^{N} \frac{\mu_{i} p_{\mathrm{r}} \min \left(1, \hat{d}^{-\alpha}\right)}{p_{\mathrm{r}} \min \left(1, \hat{d}^{-\alpha}\right)-p_{i} \min \left(1, d^{-\alpha}\right)} \exp \left[-\frac{\gamma_{\mathrm{t}} N_{0}}{p_{i} \min \left(1, \tilde{d}^{-\alpha}\right)}-\frac{\gamma_{\mathrm{t}} N_{0}}{p_{\mathrm{r}} \min \left(1, \hat{d}^{-\alpha}\right)}\right] \\
& \times\left\{\mathbb{E}\left\{\exp \left[-\frac{\gamma_{\mathrm{t}} \mathcal{I}_{s r}}{p_{i} \min \left(1, \tilde{d}^{-\alpha}\right)}-\frac{\gamma_{\mathrm{t}} \mathcal{I}_{o}}{p_{\mathrm{r}} \min \left(1, \hat{d}^{-\alpha}\right)}\right]\right\}-\exp \left[\frac{\gamma_{\mathrm{t}} N_{0}}{p_{\mathrm{r}} \min \left(1, \hat{d}^{-\alpha}\right)}\right]\right. \\
& \left.\times \exp \left[-\frac{\gamma_{\mathrm{t}} N_{0}}{p_{i} \min \left(1, d^{-\alpha}\right)}\right] \mathbb{E}\left\{\exp \left[-\frac{\gamma_{\mathrm{t}} \mathcal{I}_{s r}}{p_{i} \min \left(1, \tilde{d}^{-\alpha}\right)}-\frac{\gamma_{\mathrm{t}} \mathcal{I}_{o}}{p_{i} \min \left(1, d^{-\alpha}\right)}\right]\right\}\right\},
\end{aligned}
$$

where there are two expectation terms remained after taking the expectation over the discrete power levels of the typical source. The first expectation of (60) can be derived according to (44) by replacing $p_{i} \min \left(1, d^{-\alpha}\right)$ with $p_{\mathrm{r}} \min \left(1, \hat{d}^{-\alpha}\right)$. The second expectation of (60) has been derived in (44).

Next, we will derive a lower bound of the approximate success probability when the relay is activated for the data retransmission. Denoted as $\check{P}_{\text {suc }}^{\mathrm{c}}$, the success probability lower bound is derived by replacing $\mathcal{I}_{o}$ with $\tilde{\mathcal{I}}_{o}$ in the third term of (59) for the MRC detection, then we have:

$$
\begin{aligned}
\check{P}_{\text {suc4 }}^{\mathrm{c}} & =\mathbb{E}\left\{\frac{p_{\mathrm{r}} \min \left(1, \hat{d}^{-\alpha}\right)}{p_{\mathrm{r}} \min \left(1, \hat{d}^{-\alpha}\right)-p_{o} \min \left(1, d^{-\alpha}\right)} \exp \left[-\frac{\gamma_{\mathrm{t}}\left(\mathcal{I}_{s r}+N_{0}\right)}{p_{o} \min \left(1, \tilde{d}^{-\alpha}\right)}\right]\right. \\
& \left.\times \exp \left[-\frac{\gamma_{\mathrm{t}}\left(\tilde{\mathcal{I}}_{o}+N_{0}\right)}{p_{\mathrm{r}} \min \left(1, \hat{d}^{-\alpha}\right)}\right]\left\{1-\exp \left[\frac{\gamma_{\mathrm{t}}\left(\mathcal{I}_{o}+N_{0}\right)}{p_{\mathrm{r}} \min \left(1, \hat{d}^{-\alpha}\right)}-\frac{\gamma_{\mathrm{t}}\left(\mathcal{I}_{o}+N_{0}\right)}{p_{o} \min \left(1, d^{-\alpha}\right)}\right]\right\}\right\} \\
= & \sum_{i=0}^{N} \frac{\mu_{i} p_{\mathrm{r}} \min \left(1, \hat{d}^{-\alpha}\right)}{p_{\mathrm{r}} \min \left(1, \hat{d}^{-\alpha}\right)-p_{i} \min \left(1, d^{-\alpha}\right)} \exp \left[-\frac{\gamma_{\mathrm{t}} N_{0}}{p_{i} \min \left(1, \tilde{d}^{-\alpha}\right)}\right] \\
& \times \exp \left[-\frac{\gamma_{\mathrm{t}} N_{0}}{p_{\mathrm{r}} \min \left(1, \hat{d}^{-\alpha}\right)}\right]\left\{\mathbb{E}\left\{\exp \left[-\frac{\gamma_{\mathrm{t}} \mathcal{I}_{s r}}{p_{i} \min \left(1, \tilde{d}^{-\alpha}\right)}-\frac{\gamma_{\mathrm{t}} \tilde{\mathcal{I}}_{o}}{p_{\mathrm{r}} \min \left(1, \hat{d}^{-\alpha}\right)}\right]\right\}\right. \\
& -\exp \left[\frac{\gamma_{\mathrm{t}} N_{0}}{p_{\mathrm{r}} \min \left(1, \hat{d}^{-\alpha}\right)}-\frac{\gamma_{\mathrm{t}} N_{0}}{p_{i} \min \left(1, d^{-\alpha}\right)}\right] \\
& \times \underbrace{\left.\mathcal{W}_{i}\right)}_{\mathcal{E}\left\{\exp \left[-\frac{\gamma_{\mathrm{t}} \mathcal{I}_{s r}}{p_{i} \min \left(1, \tilde{d}^{-\alpha}\right)}-\frac{\gamma_{\mathrm{t}}\left(\tilde{\mathcal{I}}_{o}-\mathcal{I}_{o}\right)}{p_{\mathrm{r}} \min \left(1, \hat{d}^{-\alpha}\right)}-\frac{\gamma_{\mathrm{t}} \mathcal{I}_{o}}{p_{i} \min \left(1, d^{-\alpha}\right)}\right]\right\}}],
\end{aligned}
$$

where there are two expectation terms. The first expectation of (61) can be derived according to (54) by replacing
$2 p_{i} \min \left(1, d^{-\alpha}\right)$ with $p_{\mathrm{r}} \min \left(1, \hat{d}^{-\alpha}\right)$. The second expectation of (61) can be derived as follows, i.e.:

$$
\begin{aligned}
\mathcal{W}\left(p_{i}\right)= & \mathbb{E}_{\Pi_{\mathrm{s}}}\left\{\prod _ { x \in \Pi _ { \mathrm { s } } } \mathbb { E } _ { p _ { x } , G _ { x } , G _ { x r } , G _ { x r o } , \mathcal { R } } \left\{\operatorname { e x p } \left[-\frac{\gamma_{\mathrm{t}} p_{x} G_{x r} \min \left(1, \ell_{x}^{-\alpha}\right)}{p_{i} \min \left(1, \tilde{d}^{-\alpha}\right)}\right.\right.\right. \\
& \left.\left.\left.-\frac{\gamma_{\mathrm{t}} \mathbf{1}\left(\gamma_{x}<\gamma_{\mathrm{t}}, \gamma_{x r} \geq \gamma_{\mathrm{t}}\right) p_{\mathrm{r}} G_{x r o} \min \left(1, \ell_{x}^{-\alpha}\right)}{p_{\mathrm{r}} \min \left(1, \hat{d}^{-\alpha}\right)}-\frac{\gamma_{\mathrm{t}} p_{x} G_{x} \min \left(1, \ell_{x}-\alpha\right)}{p_{i} \min \left(1, d^{-\alpha}\right)}\right]\right\}\right\} \\
\approx & \mathbb{E}_{\Pi_{\mathrm{s}}}\left\{\prod _ { x \in \Pi _ { \mathrm { s } } } \mathbb { E } _ { p _ { x } , G _ { x } , G _ { x r } , G _ { x x o } } \left\{(1-\zeta) \exp \left[-\frac{\gamma_{\mathrm{t}} p_{x} G_{x r} \min \left(1, \ell_{x}^{-\alpha}\right)}{p_{i} \min \left(1, \tilde{d}^{-\alpha}\right)}\right]\right.\right. \\
& \times \exp \left[-\frac{\gamma_{\mathrm{t}} p_{x} G_{x} \min \left(1, \ell_{x}^{-\alpha}\right)}{p_{i} \min \left(1, d^{-\alpha}\right)}\right]+\zeta \exp \left[-\frac{\gamma_{\mathrm{t}} p_{x} G_{x r} \min \left(1, \ell_{x}^{-\alpha}\right)}{p_{i} \min \left(1, \tilde{d}^{-\alpha}\right)}\right] \\
& \left.\left.\times \exp \left[-\frac{\gamma_{\mathrm{t}} p_{\mathrm{r}} G_{x r o} \min \left(1, \ell_{x}^{-\alpha}\right)}{p_{\mathrm{r}} \min \left(1, \hat{d}^{-\alpha}\right)}-\frac{\gamma_{\mathrm{t}} p_{x} G_{x} \min \left(1, \ell_{x}^{-\alpha}\right)}{p_{i} \min \left(1, d^{-\alpha}\right)}\right]\right\}\right\} \\
= & \prod_{j=0}^{N} \exp \left\{-\pi \lambda_{\mathrm{s}} \mu_{j}\left\{1-\left[(1-\zeta)+\frac{\zeta \min \left(1, \hat{d}^{-\alpha}\right)}{\min \left(1, \hat{d}^{-\alpha}\right)+\gamma_{\mathrm{t}}}\right]\right.\right. \\
& \left.\left.\times \frac{p_{i}^{2} \min \left(1, \tilde{d}^{-\alpha}\right) \min \left(1, d^{-\alpha}\right)}{\left[p_{i} \min \left(1, \tilde{d}^{-\alpha}\right)+\gamma_{\mathrm{t}} p_{j}\right]\left[p_{i} \min \left(1, d^{-\alpha}\right)+\gamma_{\mathrm{t}} p_{j}\right]}+2 \mathcal{Y}\left(p_{i}, p_{j}\right)\right\}\right\},
\end{aligned}
$$

where in the derivation, we first take the expectation over the event $\mathcal{R}$ using the average probability of relay being active, then the expectations are taken over the independent channel fading variables, and finally the expectation is taken over the discrete power and point process using the PGFL. The intermediate function of (62) is defined as:

$$
\begin{aligned}
\mathcal{Y}\left(p_{i}, p_{j}\right)= & \int_{1}^{\infty}\left\{1-\left[(1-\zeta)+\frac{\zeta \min \left(1, \hat{d}^{-\alpha}\right)}{\min \left(1, \hat{d}^{-\alpha}\right)+\gamma_{\mathrm{t}} \ell^{-\alpha}}\right]\right. \\
& \left.\times \frac{p_{i}^{2} \min \left(1, \tilde{d}^{-\alpha}\right) \min \left(1, d^{-\alpha}\right)}{\left[p_{i} \min \left(1, \tilde{d}^{-\alpha}\right)+\gamma_{\mathrm{t}} p_{j} \ell^{-\alpha}\right]\left[p_{i} \min \left(1, d^{-\alpha}\right)+\gamma_{\mathrm{t}} p_{j} \ell^{-\alpha}\right]}\right\} \ell \mathrm{d} \ell .
\end{aligned}
$$

It can be seen that the intermediate function $\mathcal{Y}\left(p_{i}, p_{j}\right)$ is a one-dimensional integral, and it can be numerically calculated over a finite interval of $\ell$.

\subsection{Achievable area throughput}

After deriving the success probabilities of data transmission and retransmission in the two successive subperiods over the cooperative link, we can define in this subsection the achievable area throughput of the cooperative network. We have totally four cases for the cooperative data transmission: 1) the data transmissions in both the first and second subperiods are successful; 2) the data transmission succeeds in the first subperiod, but it fails in the second subperiod; 3) the data transmission in the first subperiod is unsuccessful, and the source successfully 
retransmits the data; and 4) the data transmission in the first subperiod is unsuccessful, but the relay has the correct data copy and the relay successfully retransmits the data. Corresponding to these four cases, the maximal area throughput can be derived as:

$$
\begin{aligned}
\mathcal{T}_{\mathrm{c}} & =\max _{\tau} \lambda_{\mathrm{s}} R\left[P_{\mathrm{suc} 2}^{\mathrm{c}}+\frac{P_{\mathrm{suc} 1}^{\mathrm{c}}-P_{\mathrm{suc} 2}^{\mathrm{c}}}{2}+\frac{P_{\mathrm{suc} 3}^{\mathrm{c}}}{2}+\frac{P_{\mathrm{suc} 4}^{\mathrm{c}}}{2}\right] \\
& =\max _{\tau} \lambda_{\mathrm{s}} \frac{R}{2}\left(P_{\mathrm{suc} 1}^{\mathrm{c}}+P_{\mathrm{suc} 2}^{\mathrm{c}}+P_{\mathrm{suc} 3}^{\mathrm{c}}+P_{\mathrm{suc} 4}^{\mathrm{c}}\right),
\end{aligned}
$$

where the time allocation factor $\tau$ has the value in $(0,1)$. Since the success probabilities are rather complex with regards to $\tau$, we cannot give the closed-form solutions. Instead, we can perform the one-dimensional search to find the optimal value of $\tau$. The distance between the source and the relay is given as $\tilde{d}=\beta d$ and the distance between the relay and the destination is given as $\hat{d}=(1-\beta) d$.

Figure 6 shows the area throughput of the cooperative system with respect to the time fraction $\tau$ for different source-relay distance factors $\beta$. The transmission rate is fixed as $R=1.0 \mathrm{bits} / \mathrm{s} / \mathrm{Hz}$, and the data transmission power of the relay is fixed as $p_{\mathrm{r}}=0.1 \mathrm{~W}$. It can be seen that, with the increase of $\tau$, the system performance improves first and then deteriorates. In each time block, when the EH time gets longer, more energy can be harvested, but less time is remained for the data transmission. Therefore, the maximal area throughput is achieved as a trade-off between the $\mathrm{EH}$ amount and the data transmission duration. With the increase of $\beta$, the system performance becomes worse. When $\beta$ is small, the distance between the source and the relay is short, so the WET towards the source is of high efficiency and the data transmission towards the relay has a good success probability. In this case, a higher transmission power can be adopted by the source, and it is more likely for the relay to correctly receive the source data. As a result, the relay can more likely assist the source for the data retransmission once the original transmission fails. Moreover, our derived lower and upper approximations are tight to each other in the low regime of $\tau$, while the gap is also small in the high regime of $\tau$.

\section{Numerical and simulation results}

In this section, we first perform the Monte Carlo simulations to verify the tightness of our theoretical analysis of the success probabilities for the non-cooperative and cooperative systems analyzed in Sections 4 and 5.

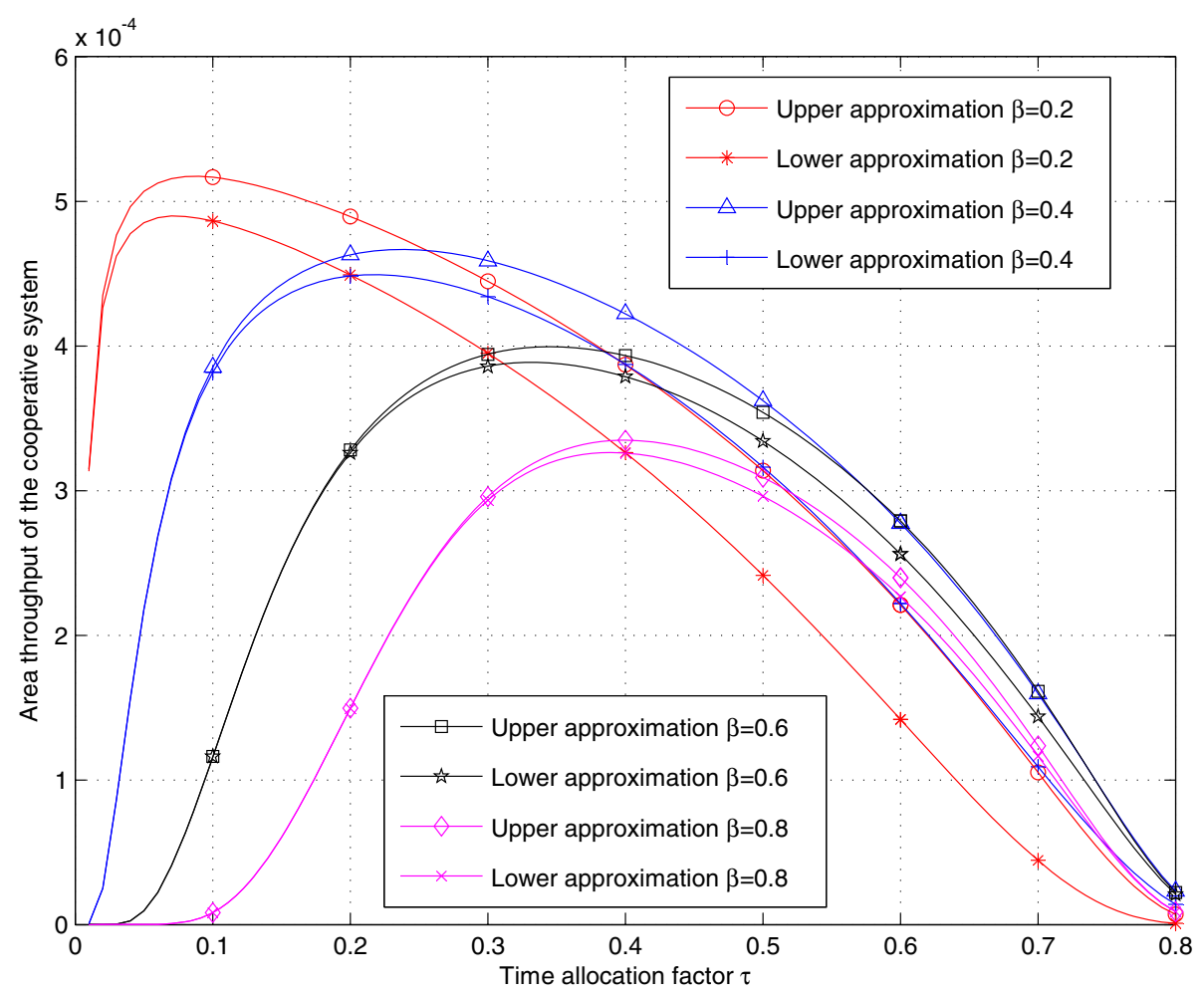

Figure 6 Area throughput of the cooperative system w.r.t. the time allocation factor $\tau$ for different location factors $\beta$. 
Then, we compare the area throughput of the noncooperative and cooperative protocols with respect to different parameter settings. Unless stated otherwise, some system parameters are set as follows: the path-loss exponent is $\alpha=3$, the noise power is $N_{0}=10^{-6} \mathrm{~W}$, the power for WET is $p_{\text {es }}=15 \mathrm{~dB}$, the distance between each source and its destination is $d=10 \mathrm{~m}$, the WET efficiency is $\eta=0.8$, the number of power level is $N=5$, the density of active source links is $\lambda_{\mathrm{s}}=10^{-3}$, the peak data transmission power is $\hat{p}=0.1 \mathrm{~W}$, the data transmission power of each relay is $p_{\mathrm{r}}=0.1 \mathrm{~W}$, the circuit power consumption is $p_{\mathrm{c}}=0.01 \mathrm{~W}$, and the transmission rate is $R=1.0$ bits/s/Hz.

\subsection{Link average throughput}

The link throughput of the non-cooperative system can be given as $\mathcal{L}_{\mathrm{n}}=R\left(P_{\text {suc } 1}^{\mathrm{n}}+P_{\text {suc } 2}^{\mathrm{n}} / 2\right)$, where the success probabilities $P_{\text {suc1 }}^{\mathrm{n}}$ and $P_{\text {suc2 } 2}^{\mathrm{n}}$ have be obtained in (31) and (35), respectively. Figure 7 shows the average link throughput $\mathcal{L}_{\mathrm{n}}$ with respect to the density of sources $\lambda_{\mathrm{s}}$ for different $\mathrm{EH}$ time fractions $\tau$. It can be seen that, the average link throughput deteriorates with the increase of the source density, because more active links will introduce more interference which can inevitably degrade the data success probability. In the lower regime of $\lambda_{s}$, the more time allocated to the WET, the better performance can be achieved. For the lower source density $\lambda_{\mathrm{s}}$, the interference is relatively weak and more energy can be harvested with higher time fraction $\tau$ to combat the detrimental effect of noise. With more energy harvested, there are also more opportunities for each source to be active for the data transmission. However, in the higher regime of $\lambda_{s}$, the system performance gets better with less time allocated to the WET. For the higher source density $\lambda_{s}$, the interference dominates over the noise, and the more time allocated to the WET, the less time is retained for the data transmission which is not helpful to improve the link throughput. The theoretical and simulation results agree well with each other, which can validate our theoretical analysis in Section 4.

The average throughput of the cooperative link is obtained as $\mathcal{L}_{\mathrm{c}}=R\left(P_{\text {suc2 }}^{\mathrm{c}}+\frac{P_{\text {suc1 }}^{\mathrm{c}}-P_{\text {suc2 } 2}^{\mathrm{c}}}{2}+\frac{P_{\text {suc3 }}^{\mathrm{c}}}{2}+\frac{P_{\text {suc } 4}^{\mathrm{c}}}{2}\right)$, where the four terms represent four cases of data transmission and retransmission with or without relaying in the two subperiods as illustrated in Subsection 5.4. The upper approximation of the link throughput can be obtained by using $\hat{P}_{\text {suc } 3}^{\mathrm{c}}$ and $\hat{P}_{\text {suc } 4}^{\mathrm{c}}$ as given in (51) and (60), respectively. Similarly, the lower approximation of the link throughput can be obtained by using $\check{P}_{\text {suc3 }}^{\mathrm{c}}$ and $\check{P}_{\text {suc4 }}^{\mathrm{c}}$ as given in (52) and (61), respectively. Figure 8 shows the average throughput of the cooperative link with respect to the EH time $\tau$ for different source densities $\lambda_{s}$. It can be seen that the link throughput increases first and then decreases with more time allocated to the WET. The optimal link throughput

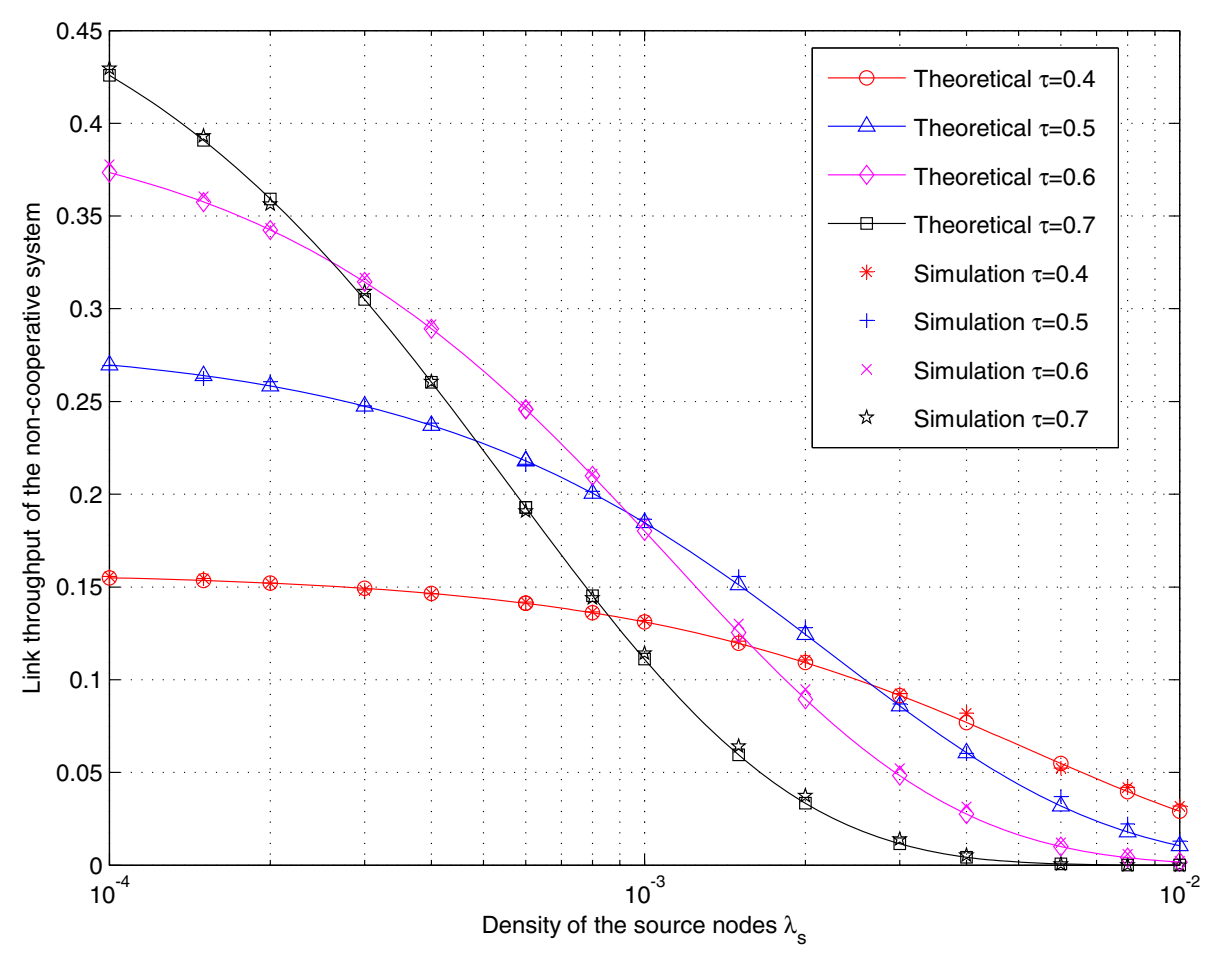

Figure 7 Link throughput of the non-cooperative system w.r.t. the density of source nodes $\lambda_{s}$ for different EH time allocations $\tau$. 


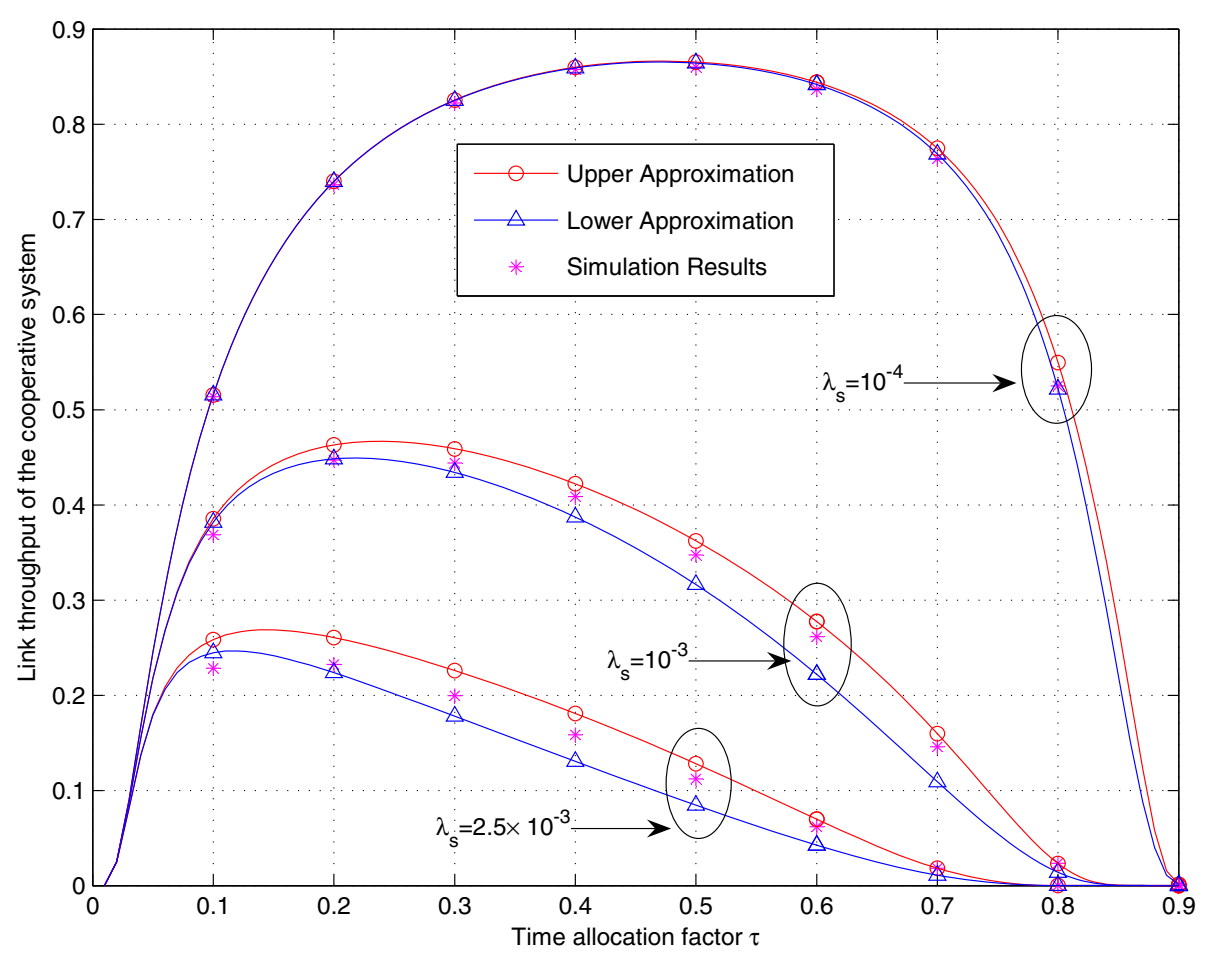

Figure 8 Link throughput of the cooperative system w.r.t. the EH time fraction $\tau$ for different source densities $\lambda_{s}$.

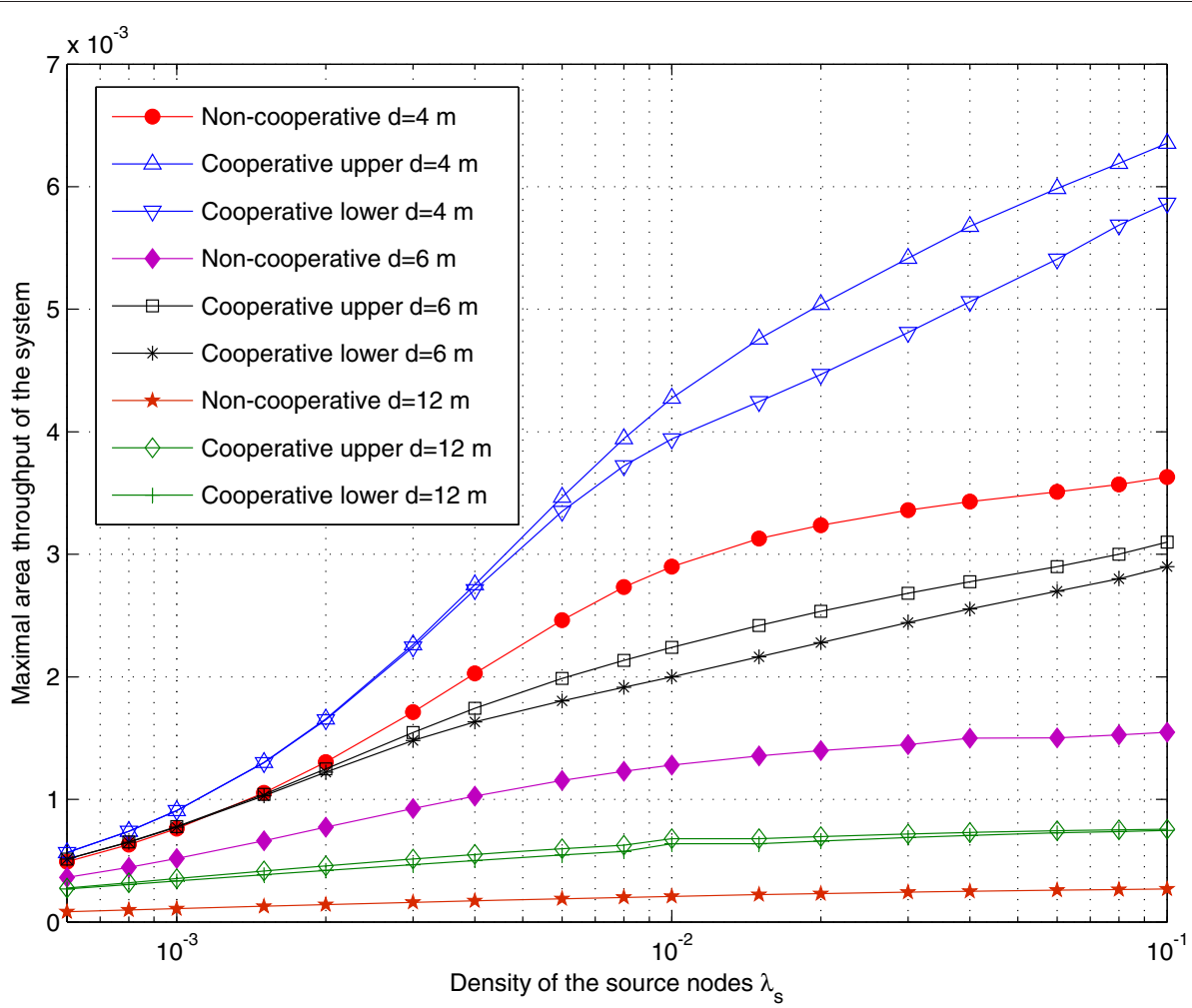

Figure 9 Maximal area throughput of the non-cooperative and cooperative systems w.r.t. the density of source nodes $\lambda_{s}$. The relative location of relay is set as $\beta=0.4$. 
represents a trade-off between the WET and the data transmission, as more energy can be harvested with more time allocation $\tau$, but less time is remained for the data transmission. When the source nodes are more densely distributed over the 2-D plane, the average throughput gets worse as more interference is imposed over each link. Moreover, the simulation results are relatively tight to the theoretical upper and lower approximations. Since several assumptions are made in the derivations, the simulation results may not strictly lie in-between the lower and upper approximations.

\subsection{Achievable area throughput}

Figure 9 shows the achievable area throughput of the noncooperative and cooperative systems with respect to the density of source nodes for different source-destination distances. The system performance improves with the increase of the source density in the studied region. However, with the increase of the distance between each source and its corresponding destination, the achievable area throughput gets smaller, because the longer the transmission distance, the lower the WET efficiency and the worse the average channel quality. The cooperative system significantly outperforms the non-cooperative system, as both the EH efficiency and data transmission robustness can be greatly improved with the assistance from the relay. For the cooperative system, the performance gap between the lower and upper approximations is small, so both of them can be effectively used to configure the system parameters.

Figure 10 shows the achievable area throughput of the non-cooperative and cooperative systems with respect to the data transmission rate for different energy transfer powers. When the transmission rate gets larger, it becomes more difficult to support the successful data transmissions due to the rigorous requirement of the channel quality. Although more outages will occur for the data transmission with a higher rate, more information can be delivered to the destination once a data transmission is successful. Affecting by such contradictory factors, as shown in the figure, the area throughput improves first and then deteriorates with the increase of the transmission rate. Moreover, there are more active communication links over the 2-D plane with a higher energy transfer power $p_{\mathrm{e}}$, which is beneficial to improve the system area throughput. The cooperative scheme can achieve a much higher area throughput than the non-cooperative scheme. For the cooperative system, both the lower and upper approximations derived in Section 5 can achieve the similar performance for various system settings.

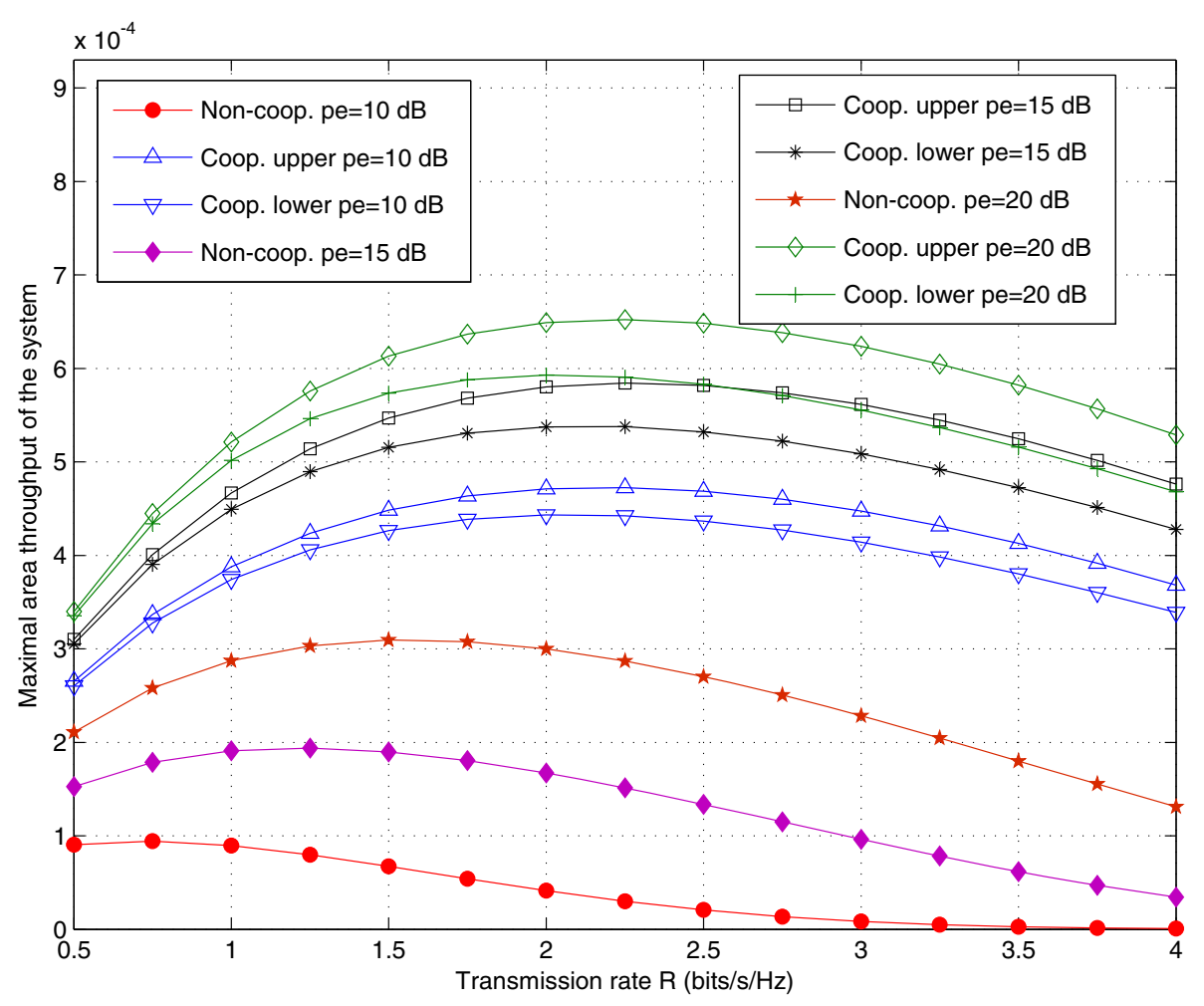

Figure 10 Maximal area throughput of the non-cooperative and cooperative systems w.r.t. the transmission rate $R$ for different energy transfer power $p_{\mathrm{e}}$. The relative location of the relay is $\beta=0.4$, and the source-destination distance is $d=10 \mathrm{~m}$. 


\section{Conclusions}

In this paper, we have proposed a framework for the cooperative wireless energy transfer and data relaying in a large-scale wireless network with the terminals randomly distributed over the plane. In each time block, only after the wireless energy transfer performed by the destination alone or together with a relay could the source transmit its data using the harvested energy. We define a set of transmission powers and analyze the probability of choosing each power level by averaging over the channel fading. Considering various transmission powers across different links, the data success probabilities are analyzed using the stochastic geometry theory. The optimal time allocation between the wireless energy transfer and the data transmission is determined through maximizing the area throughput of the non-cooperative and cooperative systems. Numerical and simulation results are provided to verify the tightness of our theoretical analysis and show the impacts of various parameter settings to the system performance. With the assistance from a relay for the wireless energy and data transmission, the cooperative scheme can significantly outperform the non-cooperative scheme.

\begin{abstract}
Abbreviations
EH: energy harvesting; ID: information decoding; RF: radio frequency; MIMO: multiple-input multiple-output; AP: access point; UT: user terminal; BS: base station; SWIPT: simultaneous wireless information and power transfer; OFDM: orthogonal frequency division multiplexing; CR: cognitive radio; WET: wireless energy transfer; DF: decode-and-forward; PPP: Poisson point process; MRC: maximal ratio combining; SINR: signal-to-interference-plus-noise ratio; 2-D: two-dimensional; PGFL: probability generating function.
\end{abstract}

\section{Competing interests}

The authors declare that they have no competing interests.

\section{Acknowledgements}

This work was supported by the China/Shandong University Postdoctoral International Exchange Program, the China Postdoctoral Science Foundation (2014M560553), the Special Funds for Postdoctoral Innovative Projects of Shandong Province (201401013), the National Natural Science Foundation of China (61371188), the Research Fund for the Doctoral Program of Higher Education (20130131110029), and the Open Research Fund of the National Mobile Communications Research Laboratory (2012D10).

Received: 17 September 2014 Accepted: 9 February 2015

Published online: 03 March 2015

\section{References}

1. A Kansal, J Hsu, S Zahedi, MB Srivastava, Power management in energy harvesting sensor networks. TransEmbedded Comput. Sys. 6(4), Artical 32 (2007)

2. J Yang, S Ulukus, Optimal packet scheduling in an energy harvesting communication system. IEEE Trans. Commun. 60(1), 220-230 (2012)

3. O Ozel, K Tutuncuoglu, J Yang, S Ulukus, A Yener, Transmission with energy harvesting nodes in fading wireless channels: Optimal policies. IEEE J. Selected Areas Commun. 29(8), 1732-1743 (2011)

4. KTutuncuoglu, A Yener, Optimum transmission policies for battery limited energy harvesting nodes. IEEE Trans. Wireless Commun. 11(3), 1180-1189 (2012)

5. X Zhou, R Zhang, C-K Ho, Wireless information and power transfer: Architecture design and rate-energy tradeoff. IEEE Trans. Commun. 61(11), 4754-4767 (2013)
6. AM Zungeru, LM Ang, S Prabaharan, KP Seng, Radio frequency energy harvesting and management for wireless sensor networks. Green Mobile Devices Netw: Energy Opt. Scav. Tech. (CRC Press, 2012). doi:10.1201/ b10081-16

7. L Liu, R Zhang, K-C Chua, Wireless information transfer with opportunistic energy harvesting. IEEE Trans. Wireless Commun. 12(1), 288-300 (2013)

8. R Zhang, CK Ho, MIMO broadcasting for simultaneous wireless information and power transfer. IEEE Trans. Wireless Commun. 12(5), 1989-2001 (2013)

9. K Huang, E Larsson, Simultaneous information and power transfer for broadband wireless systems. IEEE Trans. Signal Process. 61(23), 5972-5986 (2013)

10. H Ju, R Zhang, Throughput maximization in wireless powered communication networks. IEEE Trans. Wireless Commun. 13(1), 418-428 (2014)

11. AA Nasir, X Zhou, S Durrani, RA Kennedy, Relaying protocols for wireless energy harvesting and information processing. IEEE Trans. Wireless Commun. 12(7), 3622-3636 (2013)

12. Z Ding, SM Perlaza, I Esnaola, HV Poor, Power allocation strategies in energy harvesting wireless cooperative networks. IEEE Trans. Wireless Commun. 13(2), 846-860 (2014)

13. I Krikidis, Simultaneous information and energy transfer in large-scale networks with/without relaying. IEEE Trans. Commun. 62(3), 900-912 (2014)

14. Y Guo, J Xu, L Duan, R Zhang, Joint energy and spectrum cooperation for cellular communication systems. IEEE Trans. Commun (2014). doi: 10.1109/TCOMM.2014.2353632

15. K Huang, VKN Lau, Enabling wireless power transfer in cellular networks: Architecture, modeling and deployment. IEEE Trans. Wireless Commun. 13(2), 902-912 (2014)

16. K Huang, Spatial throughput of mobile ad hoc networks powered by energy harvesting. IEEE Trans. Inform. Theory. 59(11), 7597-7612 (2013)

17. C-h Lee, M Haenggi, Interference and outage in Poisson cognitive networks. IEEE Trans. Wireless Commun. 11(4), 1392-1401 (2012)

18. S Lee, R Zhang, K Huang, Opportunistic wireless energy harvesting in cognitive radio networks. IEEE Trans. Wireless Commun. 12(9), 4788-4799 (2013)

19. C Zhai, W Zhang, G Mao, Cooperative spectrum sharing between cellular and ad-hoc networks. IEEE Trans. Wireless Commun. 13(7), 4025-4037 (2014)

20. JN Laneman, D Tse, GW Wornell, Cooperative diversity in wireless networks: Efficient protocols and outage behavior. IEEE Trans. Infom Theory. 50(12), 3062-3080 (2004)

21. N Jindal, $S$ Weber, JG Andrews, Fractional power control for decentralized wireless networks. IEEE Trans. Wireless Commun. 7(12), 5482-5492 (2008)

22. M Haenggi, RK Ganti, Interference in large wireless networks. Foundations Trends Netw. (NOW Publishers). 3, 127-248 (2008)

23. S Cui, AJ Goldsmith, A Bahai, Energy-constrained modulation optimization. IEEE Trans. Wireless Commun. 4(5), 2349-2360 (2005)

24. JG Andrews, F Baccelli, RK Ganti, A tractable approach to coverage and rate in cellular networks. IEEE Trans. Commun. 59(11), 3122-3134 (2011)

25. A Jeffrey, D Zwillinger (eds.), Table of Integrals, Series, and Products, 7th edn (Academic Press, 2007) 